\title{
A distributed virtual MIMO coalition formation framework for energy efficient wireless networks
}

Rodrigo A Vaca Ramirez ${ }^{1 *}$, John S Thompson ${ }^{2}$, Eitan Altman ${ }^{3}$ and Victor Ramos ${ }^{4}$

\begin{abstract}
In this paper, we consider a distributed virtual multiple-input multiple-output (MIMO) coalition formation algorithm. Energy savings are obtained in the reverse link by forming multi-antenna virtual arrays for information transmission. Virtual arrays are formed by finding a stable match between two sets of single antenna devices such as mobile stations (MSs) and relay stations (RSs) based on a game theoretic approach derived from the concept of the college admissions problem. Thus, power savings are obtained through multi-antenna arrays by implementing the concepts of spatial diversity and spatial multiplexing for reverse link transmission. We focus on optimizing the overall consumed power rather than the transmitted power of MSs and RSs. Furthermore, it is shown analytically and by simulation that when the overall consumed power is considered, the energy efficiency of the single antennas devices is not always improved by forming a virtual MIMO array. Hence, single antenna devices may prefer to transmit on their own when channel conditions are favorable. In addition, the simulation results show that the framework we propose provides comparable energy savings and a lower implementation complexity when compared to a centralized exhaustive search approach.
\end{abstract}

Keywords: Game theory; Energy efficiency; Distributed decision making; MIMO

\section{Introduction}

Energy consumption has become a major research topic due to the growing energy costs which comes along with the global increase in the number of mobile subscribers. On one hand, the data volume of communication networks is expected to grow by a factor of 10 every 5 years, which brings a doubling of energy consumption over the same time period $[1,2]$. On the other hand, mobile stations' (MSs) capabilities and operation time are mostly constrained due to their limited battery resources. Most of the power expenditure of MSs in transmission mode is due to the power amplifiers and the signal processing module [3]. Thus, effective solutions allowing MSs to maximize their battery life while optimizing the overall power expenditure rather than only the transmit power are an open research field [1,4-6].

The use of multiple antennas in wireless links has emerged as an effective way to reduce the power con-

\footnotetext{
*Correspondence: rodrigo.vaca@cns.gob.mx

1 Scientific Division, Federal Police, 01110, Constituyentes 947, Mexico City, Mexico
}

Full list of author information is available at the end of the article sumption in the reverse link. It has been shown in [7] that multi-antenna systems require less transmitted power to achieve the same capacity requirements than single antenna devices. In Long-Term Evolution (LTE), a base station (BS) may support multiple antennas. However, MSs may not be equipped with more than one single antenna due to physical constraints [8,9]. Hence, implementing effective solutions allowing MSs to benefit from the advantages of multi-antenna systems without the extra burden of having multiple antennas physically present at the users' side has become a major issue for current communication systems.

Cooperative communications have recently attracted significant attention as an effective way to improve the performance of wireless networks [10,11]. By the use of cooperative techniques, wireless devices are allowed to share and use the network resources in a more efficient way $[6,10,12-16]$. As an example, the authors in [14] present a cooperative method to share the network resources and manage interference among femtocells in a distributed manner. Hence, femtocells form coalitions to improve their performance by sharing spectral resources

\section{望 Springer}


and maximizing the spatial reuse. In [16], the authors consider the consequences that arise when two multi-antenna systems share the same spectrum band. They demonstrate that if cooperation between the two systems is possible, they may achieve a performance close to the maximum sum-rate.

An important application of cooperative techniques is the formation of virtual multi-antenna arrays. In this context, a number of single antenna devices may cooperate with each other by forming virtual multiple-input multiple-output (MIMO) transmitters or receivers to reap some of the benefits of multi-antenna systems [17]. The theoretical aspects of virtual MIMO have previously been covered in $[17,18]$. Work on virtual MIMO which considers energy efficiency as an optimization metric can be found in $[8,12,19]$. The authors in $[12,19]$ illustrate the energy savings obtained when virtual MIMO techniques are used compared with non-cooperative approaches in wireless sensor networks. They argue that at certain ranges from the destination node, cooperative MIMO results in a more energy efficient solution that also reduces the total delay compared with the noncooperative case. In [8], an approach to optimize the power allocation between transmitter and relay in order to minimize the overall energy per bit consumption is presented. Moreover, it is shown that by using an optimal power allocation, the virtual MIMO case achieves an energy efficiency performance close to the ideal MIMO system.

As mentioned previously, most of the current research in energy efficient virtual MIMO tackles the problem of 'why to cooperate.' Nevertheless, there are two questions that remain unanswered, namely 'when to cooperate' and 'with whom to cooperate.' In this work, we aim to provide an answer for both questions by providing a coalition formation framework that allows single antenna devices to decide with whom to cooperate in order to obtain energy savings in the reverse link transmission.

In addition, the implementation of cooperative solutions may face many challenges due to the large scale nature of wireless systems. Cooperation comes along with costs such as power expenditure that may limit or reduce the system's performance. Moreover, if cooperation between the users is regulated by a centralized entity, a significant amount of wireless signaling overhead is required between the users and the network. Furthermore, it is well known that the use of centralized techniques entails extra implementation costs and an increase in system's complexity $[1,20,21]$. Thus, the design of effective techniques that allow the single antenna devices to autonomously decide when and with whom to cooperate with the aim of obtaining power savings in the reverse link is a matter of vital importance in communication systems $[22,23]$. In this regard, game theory provides a powerful mathematical tool for the design of distributed solutions in cooperative communications $[5,10,13,22]$. Through the use of coalitional game theory, the authors in [22] propose a merge and split distributed algorithm to form multi-antenna coalitions among single antenna devices. The aim of their work is to maximize the users' rate while accounting for the cost of cooperation in terms of power. The main difference between the merge and split scheme and our proposal is that splitting may involve finding all the possible partitions of the set formed by the users in a coalition, which increases significantly the complexity of the method when compared to the scheme presented in this paper. The authors in [24] propose a relay selection method to improve the spatial diversity in the system by using the concept of amplify and forward. They model the cooperation in wireless networks as a Stackelberg game. Moreover, they consider as optimization parameters the transmitted power and the user rate. The proposal is quite novel and interesting; however, they take into account the transmitted power rather than the overall power consumption. In addition, the communication overhead and the complexity of the method due to the use of the Stackelberg game may add an extra limitation to the system. In [5], we propose an energyefficient solution for virtual MIMO coalition formation, where cooperation is modeled as a game theoretical approach derived for the concept of stable marriage with incomplete lists. An optimal relay is selected to jointly minimize the reverse link power expenditure. Furthermore, we show that the communication overhead may be significantly reduced by using distributed techniques. Nevertheless, a major drawback of the framework proposed in [5] is that the number of elements able to join the coalition is constrained to a limited number, typically two.

The main contributions of this paper are as follows: (1) to provide a distributed low-complexity virtual MIMO coalition formation algorithm to reduce the energy consumption in the reverse link; (2) our solution can support any number of transmitters participating in the coalitions; (3) we focus on enhancing the MS performance by forming virtual coalitions with the relay stations (RSs). Moreover, we optimize the overall power consumption of MSs and RSs for reverse link transmission; (4) we analyze our proposal from both diversity and capacity perspectives; and (5) our proposal focuses on reducing the overall device consumed power rather than the transmitter radio frequency (RF) power, thus we take into account the power consumption of the RF components such as the power amplifiers and the baseband (BB) module.

The rest of the paper is structured as follows: Section 2 describes the problem scenario, Section 3 presents our power consumption model and performance measures. In 
Section 4, our cooperative framework is shown. Moreover, in Section 5, we present a theoretical analysis of the consequences arising when optimizing the overall consumed power rather than the transmitted power when implementing spatial diversity and spatial multiplexing in multi-antenna systems. A summary of the comparison schemes and our simulation scenario is described in Section 6. Simulation results are presented in Section 7. Finally, Section 8 offers concluding remarks.

\section{System scenario}

In this section, the scenario adopted in this paper is described. We consider a system with $N$ single antenna MSs transmitting data to a multi-antenna base station. In addition, $R$ single antenna RSs are uniformly distributed through the cell. We assume $R \gg N$, since a bigger number of RSs than MSs provides better chances of having an increase in power savings due to the gains in capacity and diversity that can be obtained through the formation of virtual arrays. If the number of RSs is equal or less than the number of MSs, the performance of the system will get close to a single-input multiple-output (SIMO) system since the odds to find a suitable RS to cooperate may decrease. Moreover, previous work as the one presented in [25] suggests that RSs can be also solar powered, thus scenarios where a significant amount of RSs is deployed can be used as a suitable option to reduce power consumption.

Regarding power computations, we consider the overall power expenditure of MSs and RSs for coalition formation when forming virtual arrays. In order to improve the user's performance, single antenna devices (MSs and RS) are allowed to cooperate by forming $M_{t} \times M_{r}$ virtual MIMO coalitions, where $M_{r}$ is the number of antennas at the BS, and $M_{t}$ is the number of single antenna devices forming a virtual MIMO link. If cooperation is not feasible, MSs will prefer to transmit on their own to the BS in SIMO mode.
In Figure 1, the orthogonal frequency division multiple access (OFDMA) scenario is shown. The system bandwidth $B(\mathrm{~Hz})$ is divided into $X$ resource blocks (RBs). In order to avoid mutual interference, an $\mathrm{RB}$ is assigned to each user independently. An RB defines the basic timefrequency unit with bandwidth $B_{R B}=B / X(\mathrm{~Hz})$.

\subsection{Virtual MIMO link}

Figure 2 shows a virtual $M_{t} \times M_{r}$ MIMO link implementing spatial multiplexing. At the first time slot, the MS forwards the information vector $\mathbf{s}$ to its peers by using the cooperative link. In the subsequent slot, the MS and RSs will transmit the information vector $\mathbf{s}$ at the reverse link through the MIMO channel $\mathbf{H}$. We use decode-andforward which is an obvious method for multiple transmitters cooperating to send data to a multiple antenna receiver as previously shown in [26]. In addition, to avoid mutual interference, the reverse and the cooperative link should be designed to be orthogonal to each other. When spatial multiplexing is implemented, we assume that the cooperative link has sufficient bandwidth for information transmission, thus cooperation is supported without any major interference issue. Hence, MSs can transmit their signal vector $\mathbf{s}$ to the cooperating peers and they can demultiplex it into independent information streams for simultaneous transmission in the next time slot. This adds an extra delay in the end-to-end transmission. Nevertheless, this is a common limitation of virtual MIMO cooperative approaches. A similar representation can be used for the spatial diversity concept by replacing the vector $\mathbf{s}$ with the information symbol $s$. Thereby, all antennas involved in the coalition transmit the same symbol $s$ in the reverse link.

\subsection{Cooperative link}

For the single antenna devices (MSs and RSs) to cooperate among each other, the setup and maintenance of a cooperative link is required. The cooperative link is

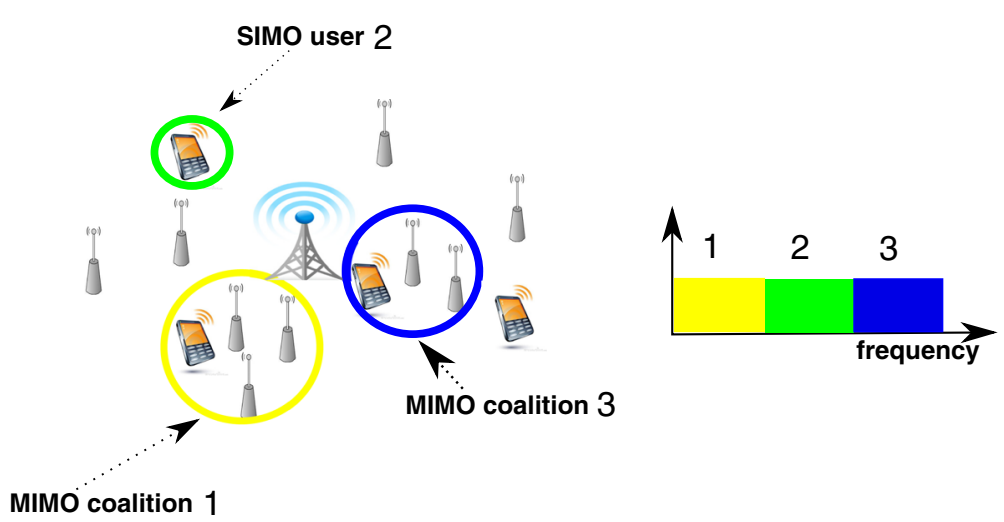

Figure 1 User cooperation example coalitions considering an OFDMA transmission model. 


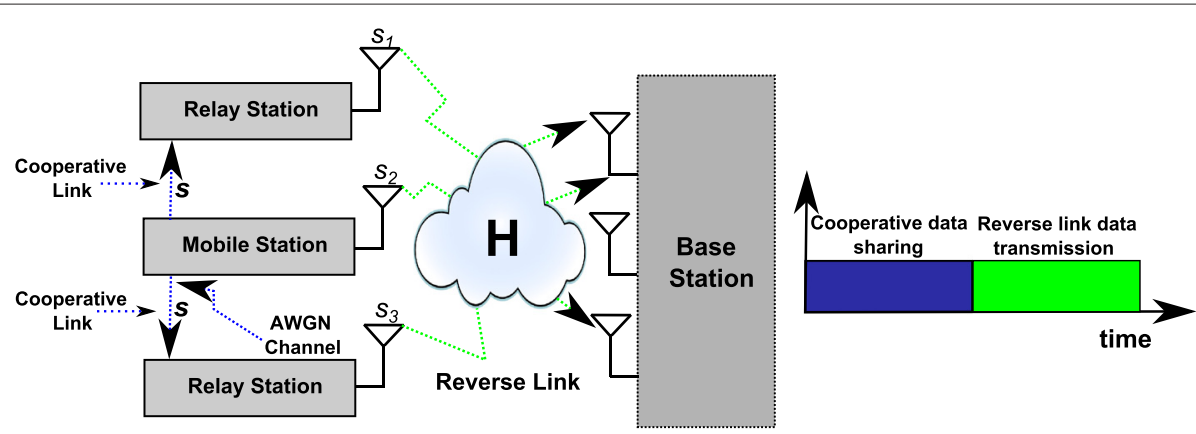

Figure 2 A virtual $M_{t} \times M_{r}$ MIMO link.

based on a short-range transmission, which is primarily used for information exchange between the transmitting peers. Thus, the channel between the MS- $n$ and the RS$r$ can be modeled as a $\kappa$ th-power path loss (loss $\left.\approx \frac{1}{l_{n r}^{\kappa}}\right)$ with additive white Gaussian noise (AWGN). Accordingly, the received power $P_{n r}$ at the RS-r, transmitted from the MS- $n$ is given by:

$$
P_{n r}=P_{t n r} l_{n r}^{-\kappa}
$$

where $l_{n r}$ is the distance between the RS- $r$ and the MS$n$, and $P_{t n r}$ is the transmitted power for cooperation. Hence, the signal-to-noise-ratio (SNR) at the RS side is represented by:

$$
\eta_{n r}=\frac{P_{n r}}{N_{0}},
$$

where $N_{0}$ is the noise power. Moreover, due to the broadcast nature of the wireless channel, when the MS broadcasts its information to the farthest RS in the coalition, all other RSs can also receive and decode simultaneously this information. Thus, define $S_{n}^{\prime} \in R$ as the subset of RSs which have formed a coalition with the MS- $n$. The cost of cooperation can be defined as the MS's maximum transmitted power to reach the farthest RS in the coalition. Thereby, define the set of distances between the MS- $n$ and its $S_{n}^{\prime}$ subset of RSs as:

$$
\begin{aligned}
D_{n r}^{*} & =\left\{l_{n(1)}, l_{n(2)}, \ldots, l_{n(\omega)}\right\}, \\
\text { s.t } l_{n(1)} & \leq l_{n(2)} \leq \ldots \leq l_{n(\omega),}
\end{aligned}
$$

where $\omega=\left|S_{n}^{\prime}\right|$ and $|$.$| define the cardinality of the sub-$ set. Thus, by using Equations 1 and 2, the power spent for cooperation may be represented by:

$$
P_{\text {tcop }}=\eta_{n(\omega)} l_{n(\omega)}^{\kappa} N_{0} \text {. }
$$

In this work, we assume that there is sufficient bandwidth to support cooperative links without any major interference.

\subsection{Reverse link channel model}

The channel coefficient between a multi-antenna BS separated by a distance $l_{k}$ from the $k$ th MIMO coalition is determined by path loss, log-normal shadowing, and channel variations caused by frequency selective fading. In this work, a fading Rayleigh channel is considered, thus the fading coefficients for an $M_{r} \times M_{t}$ MIMO channel can be represented by a matrix:

$$
\mathbf{H}=\left[\begin{array}{cccc}
h_{1,1} & h_{1,2} & \cdots & h_{1, M_{t}} \\
h_{2,1} & h_{2,2} & \cdots & h_{2, M_{t}} \\
\vdots & \vdots & \ddots & \vdots \\
h_{M_{r}, 1} & h_{M_{r}, 2} & \cdots & h_{M_{r}, M_{t}}
\end{array}\right]
$$

where each matrix element defines a zero mean circular symmetric complex gaussian (ZMCSCG) random variable with unit variance [7]. If the MS prefers to transmit in SIMO mode, the channel can be defined by the following vector:

$$
\mathbf{h}=\left[h_{1}, h_{2}, \ldots, h_{M_{r}}\right]^{T} .
$$

Furthermore, path loss and shadowing are considered to attenuate the transmitted signal, thus, the received power $P_{r}$ at the BS side is given by [27]:

$$
P_{r}=P_{t} 10^{\frac{-L\left(l_{k}\right)+X_{\sigma}}{10}},
$$

where $P_{t}$ represents the transmitted power, $X_{\sigma}$ is the lognormal shadowing value $(\mathrm{dB})$ with standard deviation $\sigma$, and $L\left(l_{k}\right)$ is the distance dependent path loss $(\mathrm{dB})$ which is calculated as follows:

$$
L\left(l_{k}\right)=a+b \log _{10}\left(l_{k}\right)[\mathrm{dB}],
$$

where $a=15.3$ and $b=37.6$ are path loss constants for a micro urban cell scenario. Moreover, since single antenna devices use a short-range transmitter for information exchange, a valid assumption is to consider that the elements involved in a MIMO coalition are sufficiently closely spaced to experience the same shadowing and 
path loss. Nevertheless, the channel fading experienced for the single antenna devices involved in a MIMO coalition can be considered as uncorrelated [28]. In addition, we assume the receiver and transmitter know the channel coefficients between them. State-of-the-art wireless standards such as LTE may implement closed-loop techniques to obtain current channel state information [29]. In this work, coalitions are formed to reduce the reverse link power consumption by using the concepts of spatial diversity and spatial multiplexing as shown below.

\subsubsection{Spatial diversity}

The received signal at the BS from the $k$ th MIMO coalition is represented as:

$$
\mathbf{y}_{k}=\sqrt{\frac{P_{r}}{M_{t}}} \mathbf{H w} s+\mathbf{n},
$$

where $M_{t}$ is the number of transmit antennas per coalition, $s$ is the scalar information symbol with unit energy, $\mathbf{n}$ is the noise and, $\mathbf{w}$ is a complex weight vector that should satisfy $\|\mathbf{w}\|_{F}^{2}=M_{t}$ to constrain the total average transmitted power, where $\|\cdot\|_{F}^{2}$ is the Frobenius norm.

Accordingly, the SNR for a MIMO coalition is given by [7]:

$$
\eta_{k_{-} \text {mimo }}=\frac{\left\|\mathbf{g}^{H} \mathbf{H w}\right\|_{F}^{2} P_{r}}{M_{t}\|\mathbf{g}\|_{F}^{2} N_{0}}
$$

where $N_{0}$ is the noise power and $\mathbf{g}$ is an $M_{r} \times 1$ complex weight vector which multiplies the received signal at the BS. Thus, maximizing the SNR at the receiver side is equivalent to maximizing the term $\left\|\mathbf{g}^{H} \mathbf{H w}\right\|_{F}^{2} /\|\mathbf{g}\|_{F}^{2}$. The proper choices of $\mathbf{w} / \sqrt{M_{t}}$ and $\mathbf{g}$ that maximize the SNR are the input and output singular value vectors corresponding to the maximum singular value $\sigma_{\max }$ of $\mathbf{H}$ [7]. By the use of the singular value decomposition (SVD) the channel matrix can be represented as $\mathbf{H}=\mathbf{U} \Sigma \mathbf{V}^{H}$, where $\mathbf{V}^{H}$ represents the conjugate transpose of $\mathbf{V}$. Moreover, the columns of $\mathbf{V}$ and $\mathbf{U}$ are known as the input and output singular vectors, respectively. In addition, $\Sigma=$ $\operatorname{diag}\left\{\sigma_{1}, \sigma_{2}, \ldots, \sigma_{J}\right\}$ with $\sigma_{i} \geq 0$, where $\sigma_{i}$ is the $i$ th singular value of the channel, and $J$ is the rank of $\mathbf{H}$. Thus, the received SNR at the BS side from the $k$ th MIMO coalition may be expressed as follows:

$$
\eta_{k_{-} \operatorname{mimo}}=\frac{\sigma_{\max }^{2} P_{r}}{N_{0}},
$$

In the case of a SIMO user, Equation 9 is re-written in the following way:

$$
\mathbf{y}_{k}=\sqrt{P_{r}} \mathbf{h} s+\mathbf{n}
$$

Thereby, the received SNR at the BS may be represented by [7]:

$$
\eta_{k_{-} \text {simo }}=\frac{\|\mathbf{h}\|_{F}^{2} P_{r}}{N_{0}},
$$

\subsubsection{Spatial multiplexing}

When channel knowledge is assumed, the individual spatial channel modes may be accessed through linear processing at the transmitter and receiver side [7]. Hence, a signal vector $\mathbf{s}$ of dimension $J \times 1$ which is transmitted from the $k$ th MIMO coalition through a rank $J$ MIMO channel, $\mathbf{H}$, after linear processing at the BS side is represented by:

$$
\begin{aligned}
\tilde{y}_{k} & =\sqrt{\frac{P_{r}}{M_{t}}} \mathbf{U}^{H} \mathbf{H V \mathbf { s }}+\mathbf{U}^{H} \mathbf{n}, \\
& =\sqrt{\frac{P_{r}}{M_{t}}} \Sigma \mathbf{s}+\tilde{\mathbf{n}},
\end{aligned}
$$

where $\mathbf{V}$ represents the matrix with dimensions $M_{t} \times J$ that multiplies $\mathbf{s}$ at the transmitter side. Moreover, $\mathbf{U}^{H}$ represents the matrix with dimensions $M_{r} \times J$ that multiplies the signal at the receiver side. In addition, $\tilde{\mathbf{n}}$ is the ZMCSCG noise vector after processing, with dimensions $J \times 1$. The transmitted signal vector $\mathbf{s}$ must satisfy $\mathbf{E}\{\mathbf{s s}\}^{H}=M_{t}$ to constrain the total transmitted power. Furthermore, Figure 3 shows how $\mathbf{H}$ is decomposed into $J$ parallel single-input single-output (SISO) channels under

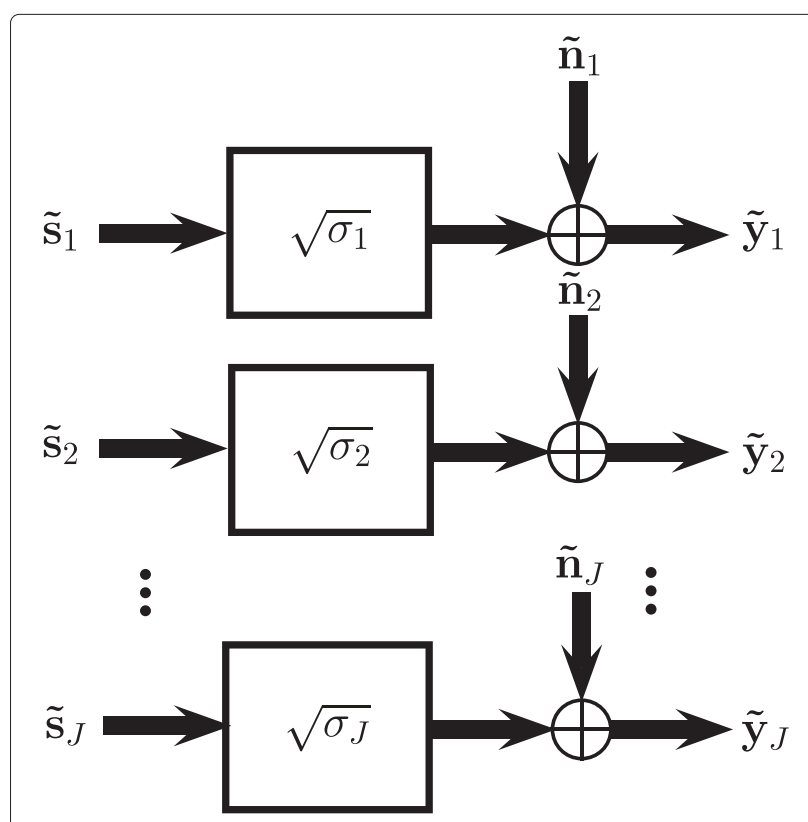

Figure 3 Modal decomposition of the channel. 
the assumption of channel knowledge at the transmitter side, where each parallel sub-channel satisfies:

$$
\tilde{y}_{i}=\sqrt{\frac{P_{r}}{M_{t}}} \sqrt{\sigma_{i}} s_{i}+\tilde{n}_{i}, \quad i=1,2, \ldots, J .
$$

Hence, the total reverse link user throughput will become the sum of the individual parallel SISO channel capacities, where the SNR of the $i$ th spatial channel (SC) is given by:

$$
\eta_{i} S C=\frac{P_{r} \zeta_{i} \sigma_{i}}{M_{t} N_{0}}
$$

where $\zeta_{i}=\mathbf{E}\left\{\left\|\mathbf{s}_{\mathbf{i}}\right\|^{2}\right\}, i=1,2, \ldots, J$, represents the transmitted power in the $i$ th SISO parallel sub-channel and must satisfy $\sum_{i=1}^{J} \zeta_{i}=M_{t}$.

Moreover, since the transmitter may access multiple parallel SISO channels, the problem becomes how to allocate the power in a way that maximizes the mutual information. The optimal value of $\zeta_{i}$ is found iteratively through the use of the water-pouring method, which is explained in detail in [30].

When cooperation is not suitable, the MSs will transmit in SIMO mode, where the achievable SNR is defined by Equation 13.

\section{Physical components power consumption model and performance metrics to optimize the overall consumed power}

In this paper, we focus on optimizing the overall power consumption of the MS's components rather than only the transmitted power. For the MIMO user case, we consider the power expenditure in both the reverse and the cooperative link. When cooperation is not feasible, MSs would prefer to transmit in SIMO mode, hence only the reverse link power expenditure is taken into account. The reverse and cooperative link power consumption mainly depend on components such as the RF parts and the BB signal processing module [3]. The RF module incorporates the power expenditure of power amplifiers, and the $\mathrm{BB}$ module comprises the power consumption for channel coding/decoding and modulation/demodulation. For modeling the RF and BB module, we use the model previously presented in [3], where the authors make an analysis of the power expenditure for both modules in a LTE mobile station. Therefore, the overall consumed power in SIMO mode, $P_{\text {simo }}$, depends primarily on the transmitted power in the reverse link $P_{t}$.

$$
P_{\text {simo }}\left(P_{t}\right)=P_{\text {circ }}\left(P_{t}\right) .
$$

Furthermore, the total consumed power to form a virtual MIMO link becomes a function of the transmitted power in the reverse link $P_{t}$, and how this is distributed between the mobile and the relay stations, which is defined by the weight vector, $\mathbf{w}$, when implementing spatial diversity and by the water filling coefficients $\zeta_{i}, i=$ $1,2, \ldots, J$, for the spatial multiplexing case. Thus, the total consumed power in the reverse link when implementing spatial diversity or spatial multiplexing respectively is obtained as follows:

$$
\begin{aligned}
& P_{\text {mimo_diversity }}\left(P_{t}\right)=\sum_{i=1}^{M_{t}} P_{\text {circ }}\left(\frac{P_{t}\left\|w_{\mathrm{i}}\right\|^{2}}{M_{t}}\right), \\
& P_{\text {mimo_capacity }}\left(P_{t}\right)=\sum_{i=1}^{M_{t}} P_{\text {circ }}\left(\frac{P_{t} \zeta_{i}}{M_{t}}\right),
\end{aligned}
$$

where $P_{\text {circ }}$ is defined as the circuit power in the reverse link spent by each of the single antenna devices such as MSs or RSs forming the MIMO link. In addition, the power expenditure due to the cooperative link, $P_{\text {circop}}$, should be added to Equations 18 and 19. Thereby, the total power expenditure to form the virtual MIMO link when implementing spatial diversity or spatial multiplexing is given by:

$$
\begin{aligned}
P_{\text {mimo_diversity_total }}\left(P_{t}, P_{\text {tcop }}\right)= & P_{\text {circop }}\left(P_{\text {tcop }}\right) \\
& +\sum_{i=1}^{M_{t}} P_{\text {circ }}\left(\frac{P_{t}\left\|w_{\mathrm{i}}\right\|^{2}}{M_{t}}\right), \\
P_{\text {mimo_capacity_total }}\left(P_{t}, P_{\text {tcop }}\right)= & P_{\text {circop }}\left(P_{\text {tcop }}\right) \\
& +\sum_{i=1}^{M_{t}} P_{\text {circ }}\left(\frac{P_{t} \zeta_{i}}{M_{t}}\right) .
\end{aligned}
$$

To model the circuit consumed power of the RF module, we consider a power amplifier array $[3,31]$ which is based on four power amplifiers: a low-power amplifier (LPA) and three high-power amplifiers, HPA 1, HPA 2, and HPA 3 , as presented in Figure 4. The power amplifier efficiency is assumed equal for both high-power amplifiers; however, HPA 1 and 2 are designed to transmit up to one fourth and to one half of the maximum transmitted power of HPA 3 , respectively. Thus, the circuit power expenditure at the reverse link $P_{\text {circ }}[\mathrm{W}]$ is given by:

$$
P_{\text {circ }}\left(P_{t}^{\star}\right)= \begin{cases}2+0.005\left(P_{t}^{\star}\right)-A & 14 \geq P_{t}^{\star} \\ \frac{1.2+0.12\left(P_{t}^{\star}\right)-\left(A-\frac{3}{4} P_{\mathrm{BB}}\right)}{4} & 17 \geq P_{t}^{\star}>14 \\ \frac{1.2+0.12\left(P_{t}^{\star}\right)-\left(A-P_{\mathrm{BB}}\right)}{2} & 20 \geq P_{t}^{\star}>17 \\ 1.2+0.12\left(P_{t}^{\star}\right)-A & 24 \geq P_{t}^{\star}>20\end{cases}
$$

where the $P_{t}^{\star}$ represents the transmitted power per antenna in $[\mathrm{dBm}]$, which is the input value converted to 


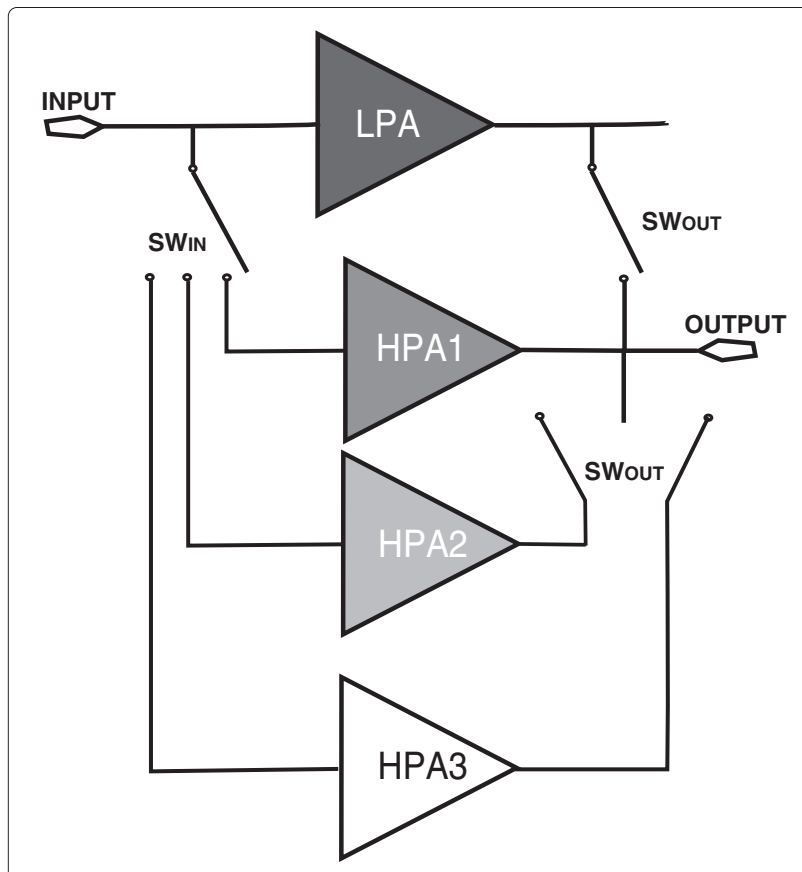

Figure 4 Internal model of the power amplifier for the RF module.

[dBm] of $P_{\text {circ }}$ in Equations 17, 20, and 21, and $A$ is a set of constant values defined as follows [3]:

$$
A=P_{T x}+P_{\text {con }}-P_{\mathrm{BB}}[\mathrm{W}] .
$$

The value $P_{T x}$ is the minimum power that the RF chain consumes in transmission mode, $P_{\text {con }}$ is the MS's power consumption when connected to the $\mathrm{BS}$, and $P_{\mathrm{BB}}$ is the power consumed by the BB module [3].

In addition, the cooperative link is constructed by using a short-range communication link, thus to model its circuit power expenditure $P_{\text {circop}}$, we use the LPA model shown below:

$$
\begin{aligned}
P_{\text {circop }}\left(P_{\text {tcop }}\right) & =2+0.005\left(P_{\text {tcop }}\right)-A[\mathrm{~W}] \\
14 & \geq P_{\text {tcop }}[\mathrm{dBm}] .
\end{aligned}
$$

\subsection{Performance metrics to optimize circuit consumed power}

The achievable throughput on the link between the $k$ th coalition and the BS when diversity is enhanced is calculated as [27]:

$$
T_{k_{-} \text {diversity }}\left(\eta_{k}\right)=n_{k}^{R B} k_{s c} \varrho_{s} \varepsilon\left(\eta_{k}\right)[\mathrm{bits} / \mathrm{s}]
$$

where $n_{k}^{\mathrm{RB}}$ is the number of resource blocks assigned to the $k$ th coalition, $k_{\mathrm{sc}}$ is the number of subcarriers per resource block, $\varrho_{s}$ is the symbol rate per subcarrier, and $\varepsilon\left(\eta_{k}\right)$ is the spectral efficiency for an LTE system [27]. Moreover, $\eta_{k}$ in Equation 25 must be replaced by Equation 11 when the user transmits in MIMO or by Equation 13 when the user transmits in SIMO mode. In the case when spatial multiplexing is implemented, the throughput is given by:

$$
T_{k_{-} \text {capacity }}\left(\eta_{i_{-} \mathrm{SC}}\right)=n_{k}^{\mathrm{RB}} k_{s c} \varrho_{s} \sum_{i=1}^{J} \varepsilon\left(\eta_{i_{-} \mathrm{SC}}\right)[\mathrm{bits} / \mathrm{s}] \text {, }
$$

where $\eta_{i \_ \text {SC }}$ is the SNR in the $i$ th individual parallel SISO channel previously given in Equation 16 and $J$ is defined as the rank of the channel. The user energy efficiency $\beta_{k}$ measures the user throughput per unit of consumed energy.

$$
\beta_{k}=T_{k} / P_{\text {total } \_k}[\text { bits } / \mathrm{J}] \text {. }
$$

This is based on the total consumed power $P_{\text {total } k \text {, }}$ where $P_{\text {total } \_k}$ is equal to $P_{\text {simo }}$ in Equation 17 when the coalition acts in SIMO mode, and to $P_{\text {mimo_diversity_total }}$ in Equation 20 or $P_{\text {mimo_capacity_total }}$ in Equation 21, when a virtual MIMO link is constructed to implement spatial diversity or spatial multiplexing, respectively. Moreover,

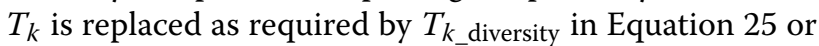
$T_{k_{-} \text {capacity }}$ in Equation 26. Additionally, the system energy efficiency $\beta_{\text {sys }}$ is defined as the ratio between the total user throughput and the total power spent by all the users in the system:

$$
\beta_{\text {sys }}=\frac{\sum_{k=1}^{N} T_{k}}{\sum_{k=1}^{N} P_{\text {total_k }}}[\mathrm{bits} / \mathrm{J}] .
$$

\section{College admission framework for distributed virtual MIMO coalition formation}

In this paper, cooperation is modeled based on the college admissions problem from game theory which has been used for decades to model and find a stable match between medical school students and residency programs in the United States [32,33]. Moreover, we use the college admission framework (CAF) to find a stable match between two sets of elements (MSs and RSs). The CAF is a generalization of the stable marriage (SM) problem, thus the solution for both problems tends to be similar in practice [34]. However, coalitions are not limited only to two participants as in the SM case [5]. As described in [32], the CAF involves a set of colleges and a set of applicants. Each applicant lists in order of preference those institutions she/he aims to attend while each institution lists in order of preference those applicants it is willing to admit. Additionally, each institution has a limit 
in the number of applicants that is able to admit. Thus, the problem becomes to assign applicants to institutions by considering both, preferences and constraints. In our problem, the $N$ MSs take the role of colleges and the $R$ RSs become the applicants. Hence, RSs are assigned to MSs to form virtual MIMO coalitions with the aim of reducing the total energy consumption of the MSs. Notice that the purpose of this work differs from well-know antennas selection techniques previously proposed in the literature [35]. In this work, by using a distributed method, we aim to find the best match between two sets of $N$ MSs and $R$ RSs rather than only find the best antenna elements to transmit/receive over a well-constrained set as previously shown in [35]. Thus, by using the CAF each MS may have multiple RS but each RS belongs to one MS only.

Virtual MIMO coalitions implement spatial diversity or spatial multiplexing respectively to obtain power savings in the reverse link. An important property of the CAF is that it leads the system to a stable solution as described in $[33,36]$. Stability means that there are no RSs and MSs in the system such that both of the following assumptions are true:

- The RS is not included into any coalition or would prefer to form a virtual MIMO link with a different MS to the one that is currently matched with;

- The MS is able to include another RS into its MIMO coalition or would prefer to cooperate with a different RS to one of its current partner RSs.

Thus, as stated in [33], when in the CAF method the conditions above are met and no match variations are presented, it always produces a stable outcome.

A mapping $M$ is a tuple of one MS with a subset of one or more RSs, such that each single antenna device (MS or RS) belongs exactly to one tuple. Hence, if $\left(n, S_{n}^{\prime}\right) \in M$, we say that the subset $S_{n}^{\prime}$ of RSs is the cooperative partner set of MS- $n$ in $M$ and vice versa, where $S_{n}^{\prime} \in R$. The distributed coalition formation algorithm is described as follows:

1. At the beginning of the algorithm, each MS in the system sends a broadcast message through the cooperative link to find the subset of RSs willing to cooperate and form a virtual MIMO link, which for the MS- $n$ is denoted by $S_{n} \in R$.

2. Moreover, the RSs in the system exchange their channel statistics in the reverse link (fading coefficient, path loss, and shadowing) and the channel statistics in the cooperative link (path loss) with the subset of MSs willing to cooperate with them, which for the RS-r is denoted by $S_{r} \in N$. Thereafter, each mobile station has the means to rank its subset of suitable RSs, $S_{n}$, by using the following utility function, that in the diversity enhancement case is defined by:

$$
\begin{aligned}
U_{n r_{-} \text {diversity }}\left(\eta_{\text {target }}\right)= & P_{\text {simo }}\left(\eta_{\text {target }}\right) \\
& -P_{\text {mimo_diversity }}\left(\eta_{\text {target }}\right),
\end{aligned}
$$

where $U_{n r_{-} \text {diversity }}$ represents the difference in power expenditure when the MS- $n$ transmits on its own or forms a virtual MIMO link with the RS- $r$, and $\eta_{\text {target }}$ is a fix target SNR that SIMO and MIMO coalitions aim to achieve. Thus, the higher is the value of the utility, the more MS- $n$ will be willing to form a virtual link with RS-r. Moreover, a negative value of $U_{n r_{-} \text {diversity }}$ means that forming a coalition with the RS- $r$ become less energy efficient, thus the MS will prefer to transmit in SIMO mode. In the case when implementing spatial multiplexing, each MS-n ranks each RS-r from its subset $S_{n}$ by using the following utility function:

$$
\begin{aligned}
U_{n r_{-} \text {capacity }}\left(T_{\text {target }}\right)= & P_{\text {simo }}\left(T_{\text {target }}\right) \\
& -P_{\text {mimo_capacity }}\left(T_{\text {target }}\right),
\end{aligned}
$$

where $U_{n r_{-} \text {capacity }}$ represents the difference in energy efficiency performance when the MS- $n$ transmits on its own or forms a coalition with RS- $r$, and $T_{\text {target }}$ represents a target transmission rate that both SIMO and MIMO users aim to achieve. Thus, as in the case where diversity is enhanced, the higher the value of the utility, the MS- $n$ will be more willing to form a virtual MIMO link with the RS-r. The MS-n's preference list $\iota_{n}$ is formed by evaluating the utility for each RS in $S_{n}$ with Equation 29 when diversity is enhanced or Equation 30 for the capacity enhancement case. Moreover, the RSs of the MS-n's preference list, $\iota_{n}$, must be sorted in descending order as follows:

$$
\begin{aligned}
\iota_{n} & =\left\{\mathrm{RS}_{n(h)}, \mathrm{RS}_{n(2)}, \ldots, \mathrm{RS}_{n(1)}\right\}, \\
\text { s.t } U_{n(1)} & \leq U_{n(2)} \leq \ldots \leq U_{n(h)},
\end{aligned}
$$

where $U_{n(r)}$ represents the pairwise comparisons between the MS- $n$ and the RS-r, and $h=\left|S_{n}\right|$, where |.| is defined as the cardinality of the sub-set. These are the values obtained from Equation 29 or 30 whenever the preference list is designed to implement spatial diversity or spatial multiplexing, respectively. Notice that when $U_{n(r)}$ becomes negative, the MS- $n$ will not consider the RS- $r$ for coalition formation, thus RS- $r$ will not be included in the MS-n ranking list, $\iota_{n}$.

3. Mobiles are only required to exchange their channel statistics in the reverse link with the RSs willing to 
cooperate with them. Based on this information, RSs are able to rank their subset of MSs, $S_{r}$, by using the following utility function when diversity is enhanced:

$$
\begin{aligned}
U_{r n \_ \text {diversity }}\left(\eta_{\text {target }}\right)= & P_{\text {circ }}\left(\frac{P_{t}\left(\eta_{\text {target }}\right)\left\|w_{\text {rs }}\right\|^{2}}{M_{t}}\right) \\
& -P_{\text {circ }}\left(\frac{P_{t}\left(\eta_{\text {target }}\right)\left\|w_{\text {ms }}\right\|^{2}}{M_{t}}\right) .
\end{aligned}
$$

For the capacity case, Equation 32 may be re-written as follows:

$$
\begin{aligned}
U_{r n_{-} \text {capacity }}\left(T_{\text {target }}\right)= & P_{\text {circ }}\left(\frac{P_{t}\left(T_{\text {target }}\right) \zeta_{\text {rs }}}{M_{t}}\right) \\
& -P_{\text {circ }}\left(\frac{P_{t}\left(T_{\text {target }}\right) \zeta_{\mathrm{ms}}}{M_{t}}\right) .
\end{aligned}
$$

Equations 32 and 33 represent the difference in power expenditure between the RS- $r$ and the MS- $n$ when forming a virtual MIMO link, respectively. Thus, the larger the value of the utility, the larger the power expenditure of the RS due to its better channel conditions in the reverse link when compared to the MS. Furthermore, the RS's preference list, $\iota_{r}$, is obtained by evaluating each of the elements in the $S_{r}$ subset by Equation 32 or 33 when using spatial diversity or spatial multiplexing, respectively. The elements of $\iota_{r}$ are also sorted in descending order as the $\iota_{n}$ case described previously in Equation 31 .

4. Once the preference lists for MSs and RSs are obtained, Algorithm 1 from [33] can be performed. Algorithm 1 is implemented in a distributed way with the sole participation of MSs and RSs, thus a centralized entity such as a BS is not required for the algorithm implementation. Hence, RSs and MSs exchange signaling messages through the cooperative link with the corresponding members contained in their preference lists $\iota_{r}$ and $\iota_{n}$, respectively. At the initial state, MSs transmit in SIMO mode, further, MSs and RSs aim to form virtual MIMO coalitions with the elements with the highest ranking in their preference lists. If that is not possible, they remove the element and propose or wait for a proposal of the next element in their corresponding list. When a new RS wants to join a coalition, the coalition checks to see if adding RS- $r$ to the $k$ th coalition will generate energy savings. The RS-r communicates its channel statistics in the reverse and cooperative links to the elements of the coalition. Hence, the $k$ th coalition computes the difference in energy consumption by adding RS- $r$ to the coalition which is calculated in an analogous way as presented in Equations 29 and 30 for the diversity and capacity case, respectively. If no power savings are obtained, the RS contacts the next MS on its list. The algorithm runs in an asynchronous way, thus the interaction between MSs and RSs can occur in an ad hoc fashion.

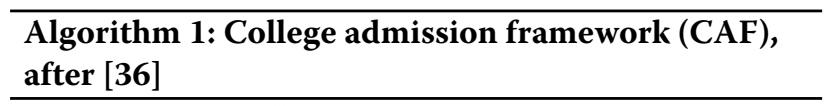

Initialization: All MSs must be operating in SIMO mode and all the RSs are free;

while There is an MS- $n$ wanting to form a MIMO link; do

' $r(h)$ is the highest ranked MS in the RS- $r$ preference list, $\iota_{r}$, to whom the RS- $r$ has not proposed yet;

if $R S-r$ is contained in the $M S_{r}(h)$ 's preference list; then

if $M S_{r}(h)$ is free; then the $M S_{r}(h)$ and the RS- $r$ become engaged; else

$M S_{r}(h)$ is already engaged with a subset of RSs, $\bar{S}_{n} \in R$

if If adding the RS-r to the $M S_{r}(h)$ current subset of $R S s, \bar{S}_{n}$, provides energy savings;

then

RS- $r$ becomes engaged; end if

if If adding the $R S$-r to the $M S_{r}(h)$ current subset of RSs, $\bar{S}_{n}$, does not provides extra energy savings. Nevertheless, $M S_{r}(h)$ prefers $R S$-r to the $R S$ - $t$ in its preference list, $\iota_{r}(h)$, where $R S-t \in \bar{S}_{n}$; then

RS- $r$ becomes engaged;

$\mathrm{RS}-t$ becomes free;

else

$M S_{r}(h)$ is deleted from the list of the RS- $r, \iota_{r}$;

end if

end if

end if

end while

\section{Analysis of the consequences in performance of MIMO systems when the overall consumed power is optimized}

In this section, a theoretical analysis is provided of the consequences arising in terms of energy efficiency when the overall power consumption is considered as an optimization metric rather than the transmitted power. Hence, to show the effects on user performance, we analytically derive the statistics of the transmitted and overall 
consumed power when implementing spatial diversity or spatial multiplexing respectively. While these statistics can be obtained experimentally, we derive them in closed form.

\subsection{Spatial diversity approach}

From Equation 7, we know that the transmitted power of any signal, $P_{t}$, can be calculated by:

$$
P_{t}=\frac{P_{r}}{10^{\frac{-L\left(l_{k}\right)+X_{\sigma}}{10}}} \text {. }
$$

Moreover, if we combine Equation 11 with Equation 34, we obtain the transmitted power for a MIMO user:

$$
P_{t \_ \text {mimo }}=\frac{\eta_{k_{-} \text {mimo }} N_{0}}{\sigma_{\max }^{2} 10^{\frac{-L\left(l_{k}\right)+X_{\sigma}}{10}}} .
$$

Given that on average, $\mathbf{E}\left\{\sigma_{\max }^{2}\right\}=M_{t} \times M_{r}$ and $X_{\sigma}=$ 0 [7], this allows us to re-write the equation above as follows:

$$
P_{t_{-} \text {mimo }}=\frac{\eta_{k_{-} \text {mimo }} N_{0}}{M_{t} \times M_{r} 10^{\frac{-L\left(l_{k}\right)}{10}}}
$$

To obtain the statistics for the transmitted power of a MIMO user, $P_{t \_ \text {mimo, }}$, we assume that the MSs are uniformly distributed in the cell. Therefore, for a circular cell of radius $R$, it is known that the probability distribution function (PDF) of the distance of any point from the center is [12]:

$$
f_{l_{k}}\left(l_{k}\right)=\frac{2 l_{k}}{R^{2}} l_{k} \in[0, R] \text {. }
$$

In addition, from Equation 8, we observe that path loss is an element depending on distance, thus to derive its PDF, we use the transformation of random variables. Thereby, we obtain the inverse relationship of the distance as a function of path loss as follows:

$$
l_{k}(L)=10^{\left(\frac{L-a}{b}\right)},
$$

Hence, the path loss $\operatorname{PDF} f_{L}(L)$ may be derived by:

$f_{L}(L)=\left|\frac{d l_{k}}{d L}\right| f_{l_{k}}\left(l_{k}(L)\right) L \in\left[-\infty, a+b \log _{10}(R)\right][d B]$,

$f_{L}(L)=\frac{2 \log (10)}{b R^{2}} 10^{\frac{2(L-a)}{b}}$.

Once the statistics for the path loss are obtained, we proceed to derive the PDF of the transmitted power. From
Equation 36, we are able to obtain the inverse relationship of the path loss as a function of the transmitted power for a MIMO user, $P_{t \_m i m o}$.

$$
L\left(P_{t_{-} \text {mimo }}\right)=-10 \log _{10}\left(\frac{\eta_{k_{-} \text {mimo }} N_{0}}{M_{t} M_{r} P_{t \_ \text {mimo }}}\right) .
$$

Thus, the PDF of the transmitted power for a MIMO user can be obtained as follows:

$$
\begin{gathered}
f_{P_{t_{-} \text {mimo }}}\left(P_{t_{-} \text {mimo }}\right)=\left|\frac{d L}{d P_{t_{-} \text {mimo }}}\right| f_{L}\left(L\left(P_{t_{-} \text {mimo }}\right)\right) \\
P_{t_{-} \text {mimo }} \in\left[0, \frac{\eta_{k_{-} \text {mimo }} \rho}{\left.M_{t} M_{r} 10^{-\left(\frac{a+b \log _{10}(R)}{10}\right)}\right],}\right. \\
f_{P_{t_{-} \text {mimo }}}\left(P_{t_{-} \text {mimo }}\right)=\frac{20}{b R^{2} P_{t_{-} \text {mimo }}} 10^{\frac{-2}{b}\left(10 \log _{10}\left(\frac{\eta_{k_{\text {mimo }} \rho} \rho}{M_{t} M_{r} P_{t_{-} \text {mimo }}}\right)+a\right) .}
\end{gathered}
$$

From Equation 22, we see that the circuit consumed power, $P_{\text {circ }}$, depends of the transmitted power when converted to $[\mathrm{dBm}]$. Thus, the inverse relationship of the transmitted power, $P_{t_{-} \text {mimo }}$, in function of the transmitted power in $[\mathrm{dBm}], P_{t_{-} \text {mimo_dBm }}$, for a MIMO user case is given by:

$$
P_{t \_ \text {mimo }}\left(P_{t \_ \text {mimo_dBm }}\right)=1 e^{-3} \times 10^{\frac{P_{t} \text { mimo d dBm }}{10}} .
$$

Thereby, the PDF of the transmitted power in $[\mathrm{dBm}]$, $P_{t \_m i m o \_d B m}$ is:

$$
\begin{gathered}
f_{P_{t} \text { mimo_dBm }}=\left|\frac{d P_{t \_ \text {mimo }}}{d P_{t \_ \text {mimo }} \mathrm{dBm}}\right| f_{P_{t} \text { mimo }}\left(P_{t \_ \text {mimo }}\left(P_{t \_ \text {mimo }} \mathrm{dBm}\right)\right) \\
P_{t \_ \text {mimo_dBm }} \in\left[-\infty, 10 \log _{10}\left(\frac{1 e^{3} \times \eta_{k_{-} \operatorname{mimo}_{0} N_{0}}}{M_{t} M_{r} 10^{-\left(a+b \log _{10}(R)\right)}}\right)\right],
\end{gathered}
$$

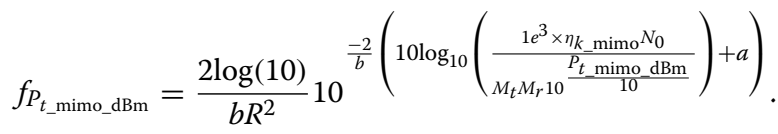

Moreover, we observe that the input of $P_{\text {circ }}$ in Equation 18 is the transmitted power for each antenna in $[\mathrm{dBm}]$. Thereby, assuming that the total transmitted power for a MIMO user, $P_{t_{-} \text {mimo, }}$ is divided evenly between each antenna as was proposed for this derivation, the PDF of the transmitted power per antenna in $[\mathrm{dBm}]$, $P_{t}^{*}$, is given by:

$$
\begin{aligned}
& \left.\left.f_{P_{t}^{*}}=\frac{2 \log (10)}{b R^{2}} 10^{\frac{-2}{b}\left(1 0 \operatorname { l o g } _ { 1 0 } \left(\frac{1 e^{3} \times \eta_{k_{-} \operatorname{mimo}} \rho}{P_{t}^{*}}\right.\right.} \frac{M_{t}^{2} M_{r} 1010}{10}\right)+a\right) \\
& P_{t}^{*} \in\left[-\infty, 10 \log _{10}\left(\frac{1 e^{3} \times \eta_{k_{\_} \text {mimo }} \rho}{M_{t}^{2} M_{r} 10^{-\left(a+b \log _{10}(R)\right)}}\right)\right] \text {, }
\end{aligned}
$$


Finally, we derive the inverse relationship of the transmitted power per antenna in $[\mathrm{dBm}]$ as a function of the circuit consumed power in the reverse link by combining Equations 18 and 22 as follows:

$$
P_{t}^{*}\left(P_{\text {mimo_diversity }}\right)= \begin{cases}\frac{\gamma+A-2}{0.005} \quad 14 \geq P_{t}^{*}, \\ \frac{4 \gamma+\left(A-\frac{3 P_{\mathrm{BB}}}{0.117}\right)-1.2}{0.17} \quad 17 \geq P_{t}^{*}>14, \\ \frac{2 \gamma+\left(A-P_{\mathrm{BB}}\right)-1.2}{0.117}, \quad 20 \geq P_{t}^{*}>17, \\ \frac{\gamma+A-1.2}{0.117} \quad 24 \geq P_{t}^{*}>20 .\end{cases}
$$

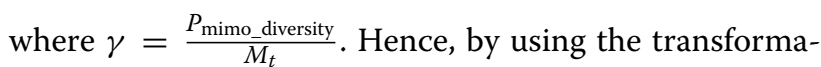
tion of random variables, the PDF of the circuit consumed power in the reverse link for a MIMO user is shown below: where $W=M_{t}^{2} M_{r}, W 1=10 \log _{10}\left(\frac{1 e^{3} \times \eta_{k_{\text {mimo }}} N_{0}}{M_{t}^{2} M_{r} 10^{-\left(a+b \log _{10}(R)\right)}}\right)$, $W 2=A-\left(0.75 \times P_{\mathrm{BB}}\right)$, and $W 3=A-P_{\mathrm{BB}}$.

Finally, by integrating the PDFs of the transmitted and circuit consumed power over their respective ranges, we obtain the cumulative distribution functions (CDFs) for transmitted and circuit consumed power, which are shown in Figures 5 and 6, respectively. In addition, we also find the CDFs by simulation to compare them with our theoretical derivations. Moreover, as an example, we consider SIMO and MIMO users with three and six antennas. Furthermore, we require the users to achieve the same target SNR, $\eta_{\text {target, }}$, whether SIMO or MIMO is used, in order to make fair comparisons in terms of power expenditure. Notice that to obtain the statistics of the overall consumed power for the SIMO case, a similar procedure is followed as the one shown for the MIMO user case. For the required values to evaluate the statistics and perform

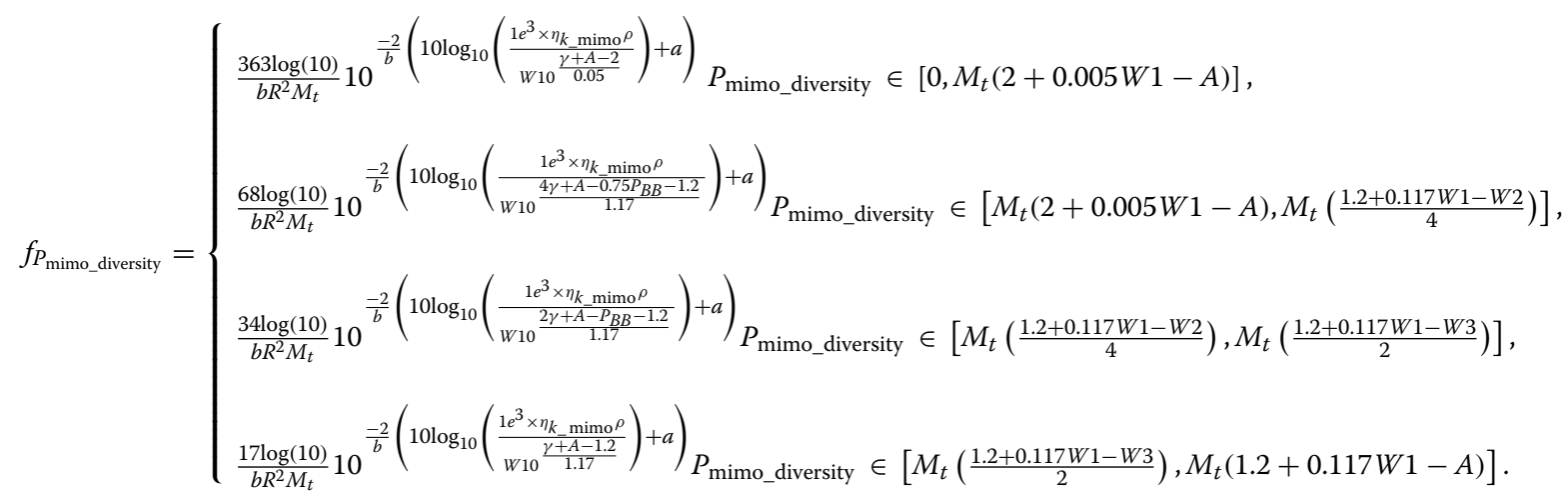

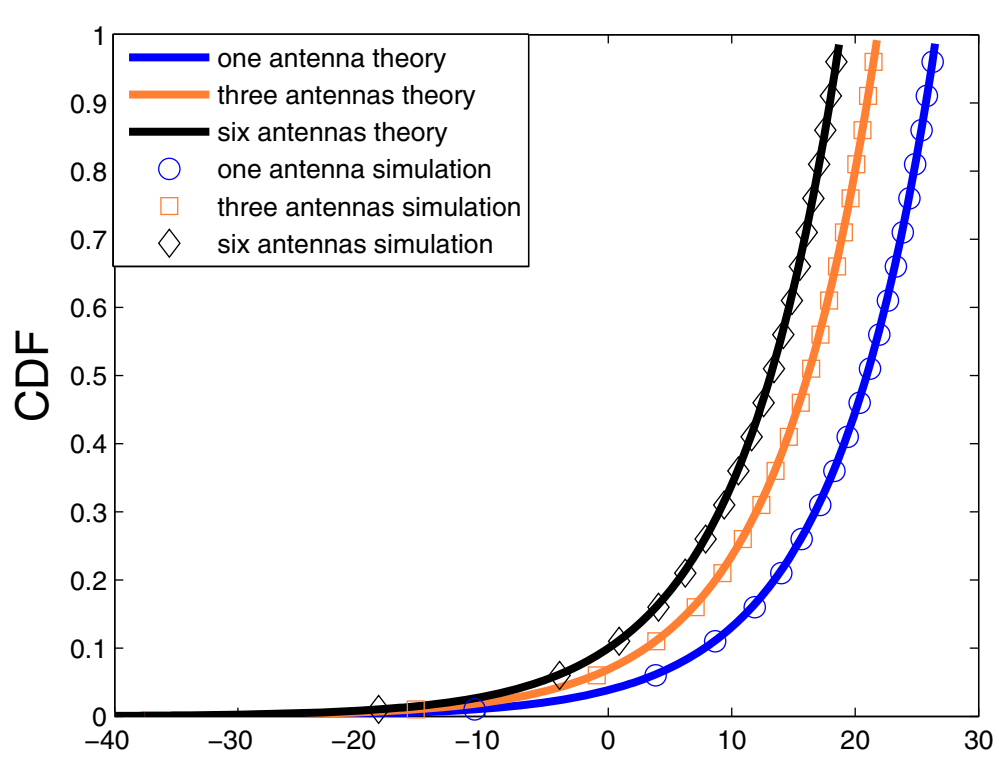

Figure $\mathbf{5}$ Transmitted power [dBm]. User performance differences, when enhancing diversity and optimizing transmitted power. 


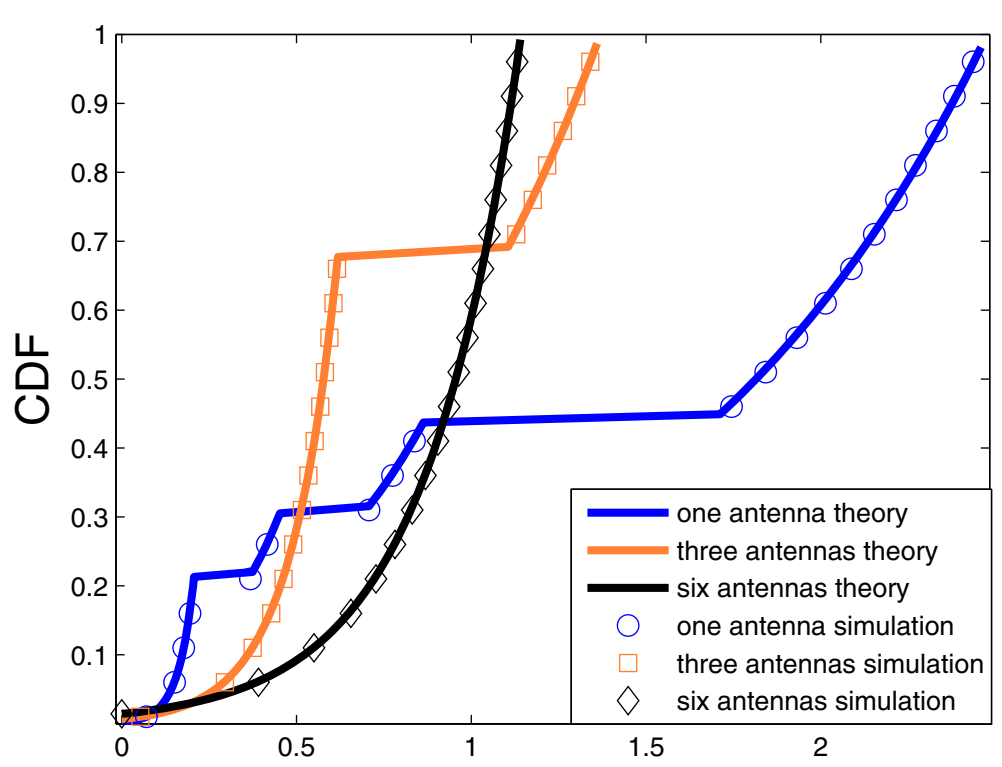

Figure 6 Overall consumed power (W). User performance differences, when enhancing diversity and optimizing overall consumed power.

the simulations, we consider the values shown in Table 1. These results are discussed further in subsection 5.3.

\subsection{Spatial multiplexing approach}

For the following derivations, we use Shannon's capacity formula for ease of analysis and without loss of generality. Thus, Equation 26 can be re-written as follows:

$$
\begin{aligned}
T_{k_{-} \text {capacity }} & =\sum_{i=1}^{J} \log _{2}\left(1+\eta_{i_{-} \mathrm{SC}}\right) \\
& =\sum_{i=1}^{J} \log _{2}\left(1+\frac{P_{r} \zeta_{i} \sigma_{i}}{M_{t} N_{0}}\right) .
\end{aligned}
$$

Moreover, if we assume equal gain conditions between the multiple parallel SISO channels $\zeta_{i}=1, \mathbf{E}\left\{\|\mathbf{H}\|_{F}^{2}\right\}=$ $M_{r} M_{t}=J \sigma_{i}$ [7], Equation 50 may be re-written as:

$$
\begin{aligned}
T_{k_{-} \text {capacity }} & =\sum_{i=1}^{J} \log _{2}\left(1+\eta_{i_{-} S C}\right) \\
& =J \log _{2}\left(1+\frac{P_{r} M_{r}}{J N_{0}}\right) .
\end{aligned}
$$

Thus, by combining Equation 34 and Equation 51, we obtain the required transmitted power as:

\begin{tabular}{|c|c|}
\hline Parameter & Value \\
\hline MSs per macro-cell, $N$ & 20 \\
\hline RSs per macro-cell, $R$ & 95 \\
\hline Number of antennas at the receiver, $M_{r}$ & 6 \\
\hline Cell radius & $150 \mathrm{~m}$ \\
\hline Number of available $\mathrm{RBs}, X$ & 20 \\
\hline Number of cells, $D$ & 1 \\
\hline Subcarriers per RB, $k_{s c}$ & 12 \\
\hline Symbol rate per subcarrier, $\varrho_{s}$ & $15 \mathrm{kbps}$ \\
\hline$P_{T x}$ & $31.8 \mathrm{dBm}$ \\
\hline$P_{\text {con }}$ & $23.8 \mathrm{dBm}$ \\
\hline$P_{B B}$ & $11.7 \mathrm{dBm}$ \\
\hline Maximum user transmit power & $24 \mathrm{dBm}$ \\
\hline Shadowing, Std. Dev., $\sigma$ & $3 \mathrm{~dB}$ \\
\hline$\eta_{\text {target }}$ & $17 \mathrm{~dB}$ \\
\hline$T_{\text {target }}$ & $910 \mathrm{kbps}$ \\
\hline$\varepsilon$ for $17 \mathrm{~dB}$ SNR & $4.5 \frac{\text { bits }}{\text { symbo }}$ \\
\hline$\kappa$ & 3.5 \\
\hline Path loss constant, $a$ & 15.3 \\
\hline Path loss constant, $b$ & 37.6 \\
\hline
\end{tabular}

$$
P_{t}=\frac{\beta}{M_{r} 10^{\frac{-L\left(d_{k}\right)}{10}}},
$$

Table 1 Simulation parameters 
where $\beta=\left(2^{\frac{T_{k_{-} \text {capacity }}}{J}}-1\right) J N_{0}$. To obtain the statistics of the transmitted power $P_{t}$, we assume that the MSs are uniformly distributed over the cell. Moreover, from Equation 52, we obtain the inverse relationship of the path loss in function of the transmitted power.

$$
L\left(P_{t}\right)=-10 \log _{10}\left(\frac{\beta}{M_{r} P_{t}}\right)
$$

Thus, by using a similar approach as in Equation 39, the PDF of the transmitted power may be obtained as:

$$
f_{P_{t}}=\frac{20}{R^{2} b P_{t}} 10^{\frac{-2}{b}\left(a+10 \log _{10}\left(\frac{\beta}{M r^{P_{t}}}\right)\right)} P_{t} \in\left[0, \frac{\beta}{\left.M_{r} 10^{-\frac{a+b \log 0_{10}^{(R)}}{10}}\right)}\right]
$$

Furthermore, we require to obtain the transmitted power in $[\mathrm{dBm}]$. Thus, the inverse relationship of the transmitted power, $P_{t}$, as function of the transmitted power in $[\mathrm{dBm}], P_{t_{-} \mathrm{dBm}}$, is given by:

$$
P_{t}\left(P_{t_{-} \mathrm{dBm}}\right)=1 e^{-3} 10^{\frac{P_{t \mathrm{dBm}}}{10}} .
$$

Thereby, by using a similar approach as in Equation 45, we derive the PDF of the transmitted power in $[\mathrm{dBm}]$ as follows:

$$
\begin{aligned}
& \left.\left.f_{P_{-\mathrm{dBm}}}=\frac{20 \log (10) 1^{-3}}{R^{2} b} 10^{\frac{-2}{b}\left(a+10 \log _{10}\left(\frac{\beta}{1 e^{-3} M_{r} 10} \frac{P_{t} \mathrm{dBm}}{10}\right)\right.}\right)\right) \\
& P_{t_{-} \mathrm{dBm}} \in\left[-\infty, 10 \log _{10}\left(\frac{1 e^{3} \times \beta}{M_{r} 10^{-\left(\frac{a+b \log _{10}(R)}{10}\right)}}\right)\right],
\end{aligned}
$$

As in the diversity case, we should observe that in order to compute the circuit consumed power $P_{\text {circ }}$, Equation 22, we require the transmitted power per antenna. Thus, assuming that the transmitted power is divided evenly over all the antennas, the PDF of the transmitted power per antenna in $[\mathrm{dBm}]$ is:

$$
\begin{aligned}
& f_{P_{t}^{*}}=\frac{20 \log (10)}{R^{2} b} 10^{\frac{-2}{b}\left(a+10 \log _{10}\left(\frac{\beta}{M_{r} M_{t} 1 e^{-3} 10 \frac{P_{t}^{*}}{10}}\right)\right)} \\
& P_{t}^{*} \in\left[-\infty, 10 \log _{10}\left(\frac{1 e^{3} \times \beta}{M_{t} M_{r} 10^{-\left(\frac{a+b \log _{10}(R)}{10}\right)}}\right)\right],
\end{aligned}
$$

Finally, we derive the inverse relationship of the transmitted power per antenna in $[\mathrm{dBm}]$ as a function of the circuit consumed power in the reverse link by combining Equations 19 and 22 as shown:

$$
P_{t}^{*}\left(P_{\text {mimo_capacity }}\right)= \begin{cases}\frac{\gamma_{1}+A-2}{0.005} \quad 14 \geq \mathrm{P}_{t}^{*}, \\ \frac{4 \gamma_{1}+\left(A-\frac{3 P_{\mathrm{BB}}}{4}\right)-1.2}{0.117} \quad 17 \geq P_{t}^{*}>14, \\ \frac{2 \gamma_{1}+\left(A-P_{\mathrm{BB}}\right)-1.2}{0.117}, \quad 20 \geq P_{t}^{*}>17, \\ \frac{\gamma_{1}+A-1.2}{0.117} \quad 24 \geq P_{t}^{*}>20 .\end{cases}
$$

where $\gamma_{1}=\frac{P_{\text {mimo_capacity }}}{M_{t}}$. Moreover, by using the transformation of random variables, the PDF of the circuit consumed power is shown below:

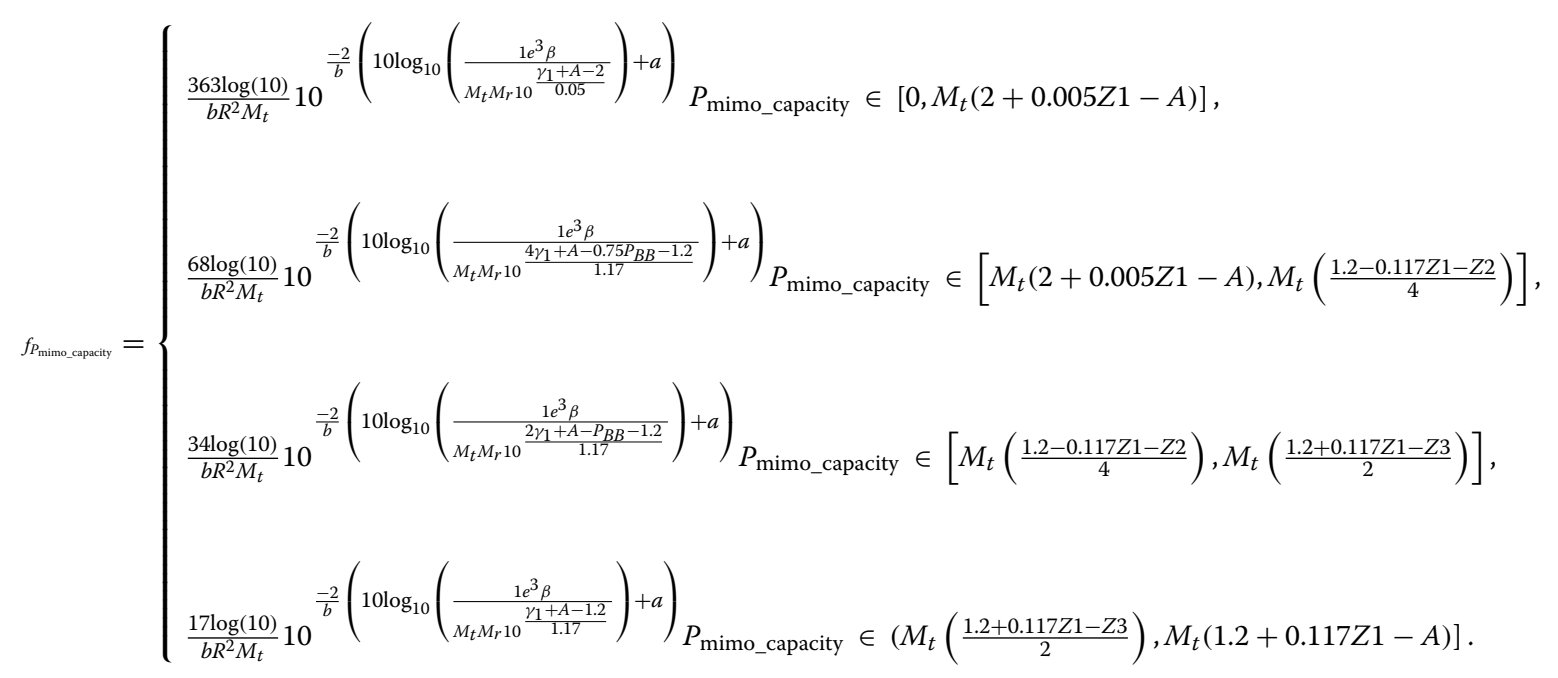


where $Z 1=10 \log _{10}\left(\frac{1 e^{3} \beta}{M_{r} M_{t} 10^{-\left(\frac{a+b l o g}{10} 0^{(R)}\right)}}\right), Z 2=A-$ $\left(0.75 \times P_{\mathrm{BB}}\right)$, and $Z 3=A-P_{\mathrm{BB}}$. Finally, as in the diversity case, by integrating the PDFs of the transmitted and circuit consumed power over their respective ranges, we obtain the CDFs for transmitted and circuit consumed power, which are shown in Figures 7 and 8, respectively. In addition, we also find the CDFs by simulation to compare them with our theoretical derivations. As an example, we consider SIMO and MIMO users carrying three and six antennas. Furthermore, we make the users independently of SIMO or MIMO to achieve the same transmission rate, $T_{\text {target }}$, in order to make fair comparisons in terms of power expenditure. To evaluate the statistics and perform the simulations, we consider the values shown in Table 1.

\subsection{Analysis}

From Figures 5 and 7, it is easy to see that increasing the number of antennas provides power savings at all percentiles of the CDF when only the transmitted power is optimized. However, this trend does not remain the same when optimizing circuit power consumption. In Figure 6, in the case of diversity, we see that the SIMO curve intersects the MIMO curves when transmitting with three and six antennas at the 30th and 45th percentile, respectively. Moreover, for the capacity case in Figure 8, we see that the SIMO curve intersects the MIMO curves when transmitting with three and six antennas at the 20th and 28th percentile, respectively. This intersection point represents that in the diversity case, SIMO is more power efficient for $30 \%$ and $45 \%$ of the users in the cell when compared to MIMO when transmitting with three and six antennas, respectively. The same relation holds for the spatial multiplexing case. This is because the MSs are able to experience better transmission conditions, when they are close to the BS. Thus, turning on the RF transmitter and the $\mathrm{BB}$ module of the relay stations is less power efficient than transmitting with only one antenna. Nevertheless, as the users get close to the cell edge increasing the number of transmit antennas tends to be an energy efficient solution when circuit power consumption is optimized. This fact can be seen from Figures 6 and 8, since as the number of antennas increases, it allows the three and six antennas curves to converge faster to the tail of the distribution. Our analysis in this section will be useful to understand the performance of our framework proposed in Section 7.

\section{Comparison schemes and simulation scenario}

To evaluate the performance of our proposal, we describe four distributed relay selection algorithms, which allow MSs and RSs to cooperate to form MIMO coalitions with the purpose of reducing the energy consumption in the reverse link. In addition, we present a baseline scheme where all MSs transmit on their own in SIMO mode. Finally, we present a centralized global optimum approach which is coordinated from the BS and based on an exhaustive search. For all the methods we describe, the communication between the MSs and RSs is made through the cooperative link. Thus, the subset of RSs willing to cooperate with the MS- $n$ is limited by the range of the cooperative link, which naturally limits the complexity of the relay selection.

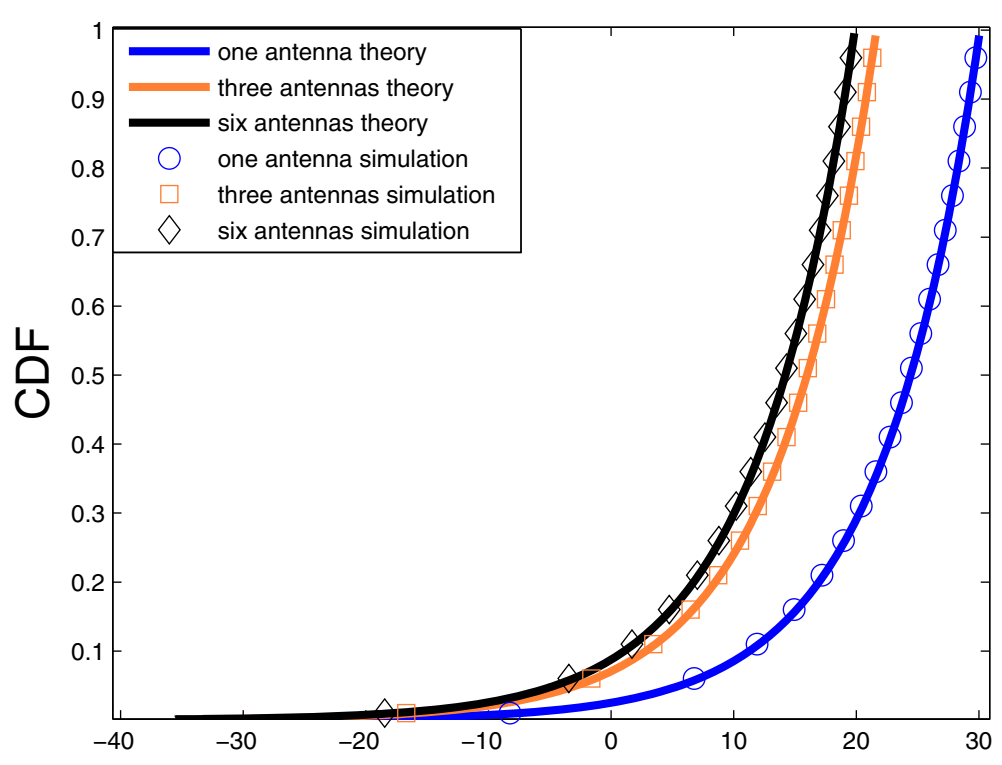

Figure 7 User performance differences, when implementing spatial multiplexing and optimizing transmitted power. 


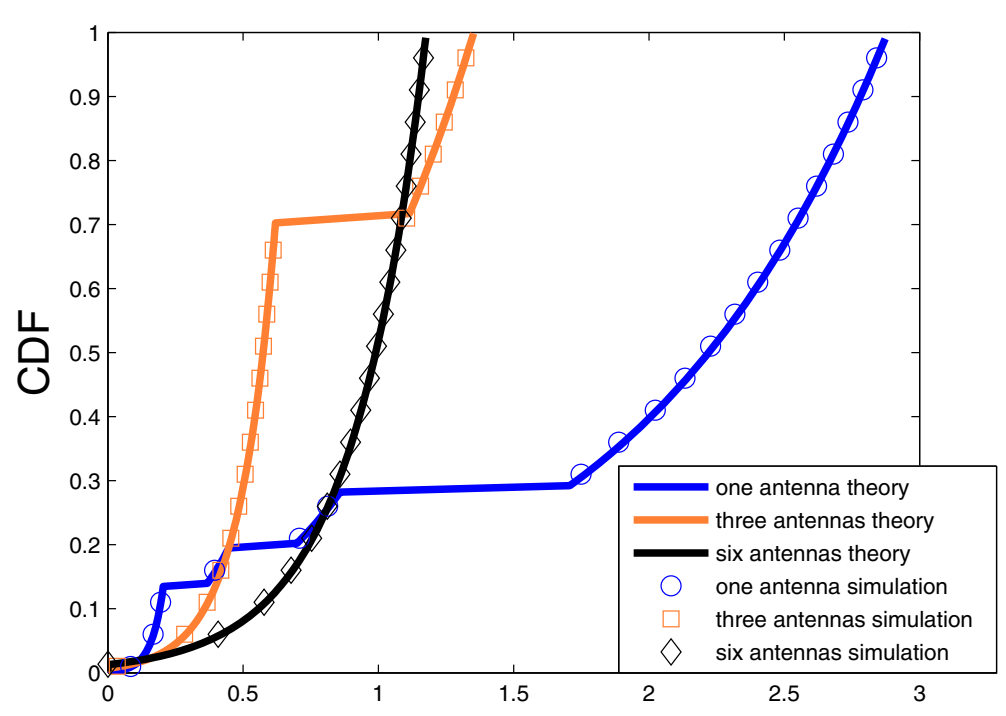

Figure 8 User performance differences, when implementing spatial multiplexing and optimizing overall consumed power.

\subsection{Minimum relaying hop (MRH) path loss selection scheme}

In [37], the authors propose a relay selection method as a function of path loss. Hence, the best RS for coalition formation is the one with the least path loss to the MS, this method always chooses the RS with the most energyefficient cooperative link.

$$
R S_{c}=\operatorname{argmin}\left\{l_{n r}^{\kappa}\right\}
$$

From Equation 60, notice that to perform the RS selection, it is just required to know the channel statistics of the cooperative link.

\subsection{Best worst (BW) channel selection scheme}

The BW method considers the quality of the cooperative and the reverse link of each RS. This is because both links have a direct influence on the total consumed energy to form the virtual MIMO link. In [37], the best worst channel is used in which the relay whose worse channel is the best is selected:

$$
\operatorname{argmin}\left\{\left\|G_{r}, \frac{1}{l_{n r}^{\kappa}}\right\|\right\}
$$

where $G_{r}=\left\|\mathbf{h}_{r}\right\|_{F}^{2} 10^{\frac{-L\left(l_{r}\right)+X_{\sigma}}{10}}$ represents the channel path gain between the RS- $r$ and the BS, and $l_{r}$ defines the distance between the $r$ th RS and the BS.

\subsection{SM scheme}

In [5], a distributed RS selection algorithm is presented which is based on the stable marriage process. This method, as in the BW channel selection scheme, requires the channel statistics from the RSs in the reverse and cooperative link plus the channel statistics of the MSs in the reverse link. Thereby, each MS and RS are able to rank their respective candidates for coalition formation. Noticed that the SM method has the same limitation as the MRH and BW methods in the sense that each MS is only able to select one RS.

\subsection{SIMO transmission}

We implement a baseline scheme, where all the MSs in the network transmit in SIMO mode.

\subsection{College admission framework scheme}

This scheme implements our RS selection method described in Section 4.

\subsection{Centralized optimum scheme}

We present a centralized global optimum scheme based on an exhaustive search approach. Thus, the BS collects the required channel statistics from RSs and MSs in order to form optimal coalitions. We implement this centralized approach with the aim of finding the price of anarchy for our proposed scheme. The price of anarchy is computed as the difference in performance between a centralized and a distributed approach [38].

\subsection{Simulation scenario}

We perform Monte Carlo simulations with Matlab ${ }^{\circ}$ using the parameters presented in Table 1. This is done to compare the performance of our method with the schemes presented above. The simulation is comprised of a single cell with the MSs and RSs distributed uniformly over 
the cell area. The cell is served by an omnidirectional BS. Moreover, the system is noise limited, hence each coalition transmits in an independent RB to avoid co-channel interference. For the case when diversity is enhanced, we assume that all the users (SIMO or MIMO) independent of their distance to the BS try to achieve the same target $\mathrm{SNR}, \eta_{\mathrm{target}}$. In the case when spatial multiplexing is used, we assume that all the users in the network aim to achieve the same data rate, $T_{\text {target }}$.

\section{Results}

From the simulations, we generate the CDFs and the graphs that illustrate the performance in terms of overall power expenditure for the schemes presented in Section 6.

When diversity is enhanced in Figure 9, we show the overall consumed power at different distances from the BS, where all the users in the cell aim to achieve the same target SNR. We observe that the distributed and centralized approaches exhibit a similar performance when compared to the baseline method at close distances from the BS (up to $75 \mathrm{~m}$ ). This is because as mentioned in the analysis presented in Section 5.1, MSs experience good transmission conditions close to the cell center. Thus, turning on the BB and the RF module of the RSs becomes less power efficient than transmitting with only one antenna. Conversely, when channel conditions are no longer so beneficial (e.g., after $75 \mathrm{~m}$ ), we see that as the MSs move away from the BS, the increase from one to a higher number of transmit antennas allows the MS to obtain potential energy savings. Furthermore, from the analysis shown in Section 5.1 and the results presented in Figure 9, we can confirm that, when circuit power consumption is optimized and spatial diversity is enhanced, by increasing the number of antennas, the obtained power savings are more visible at the cell edge than at the cell center. In Figure 10, we evaluate the system energy efficiency, given by Equation 28 . Notice that at the 50th percentile the CAF scheme is more energy efficient compared to the benchmark, the MRH path loss, the BW channel, and the SMI framework with improvements of $58 \%, 15 \%$, $10 \%$, and $5 \%$, respectively. Nevertheless, the CAF scheme has losses of $10 \%$ compared to the centralized global optimum scheme. These losses are tolerable in practice due to the significant reductions in complexity for the CAF method compared to the centralized optimum scheme: this is discussed further at the end of this section. Moreover, the better performance in energy-efficiency terms for the CAF and the centralized optimum when compared to the other distributed approaches can be easily understood as a direct consequence of the bigger number of antenna elements than can be involved in the coalition.

When spatial multiplexing is used, we aim to obtain gains in energy efficiency by dividing the total data rate requirements between the elements forming the virtual MIMO link. Thereby, as in the diversity case, we can see from Figure 11 that most of the power savings due to coalition formation are observed at the cell border. This is because, it is more power efficient to deliver high transmission rates for SIMO users close to the BS than when close to the cell edge due to the improved propagation

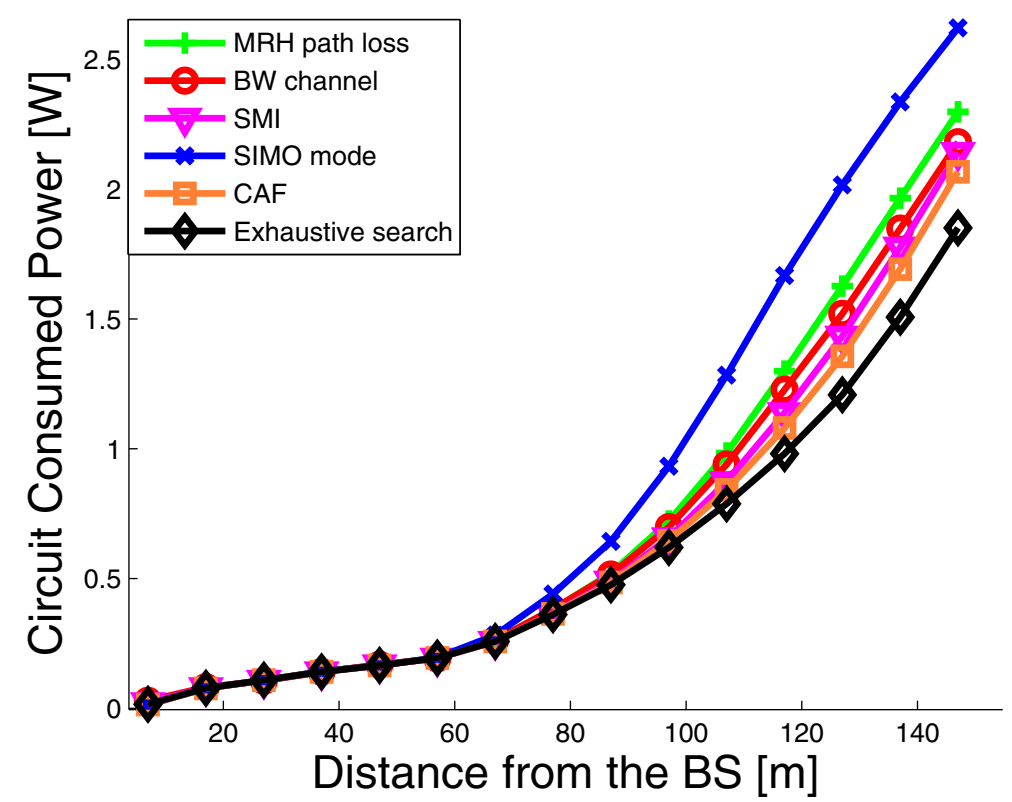

Figure 9 User circuit consumed power against distance from the BS for the SNR $=\mathbf{1 7} \mathrm{dB}$. Performance comparison when spatial diversity is implemented. 


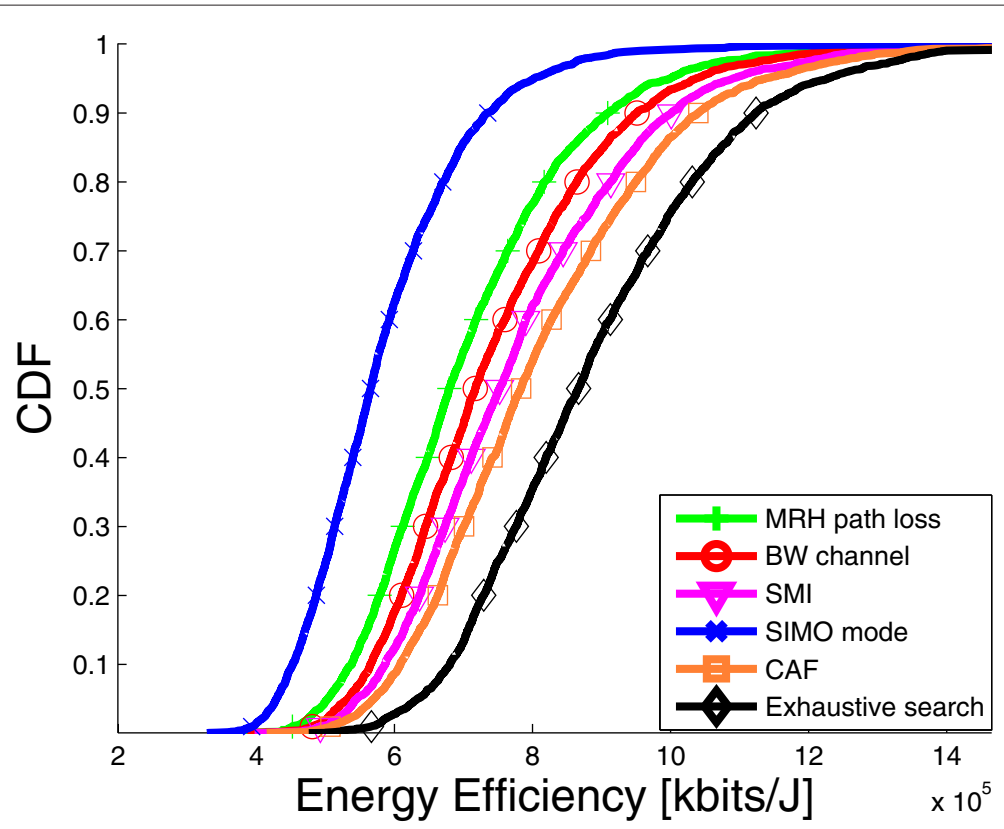

Figure 10 System CDF energy efficiency. Performance comparison when spatial diversity is implemented.

conditions. Thus, using a lower modulation order for transmitting from each antenna in a coalition when close to the cell edge becomes more power efficient than using a single transmitter. Hence, from the results obtained in Section 5.2 and Figure 11, it can be understood that increasing the number of transmit antennas to split the total rate requirement among the transmitters by implementing spatial multiplexing is more power efficient in terms of overall power consumption at the cell border than at the cell center.

Finally, in Figure 12, we show the performance in terms of energy efficiency for the approaches presented

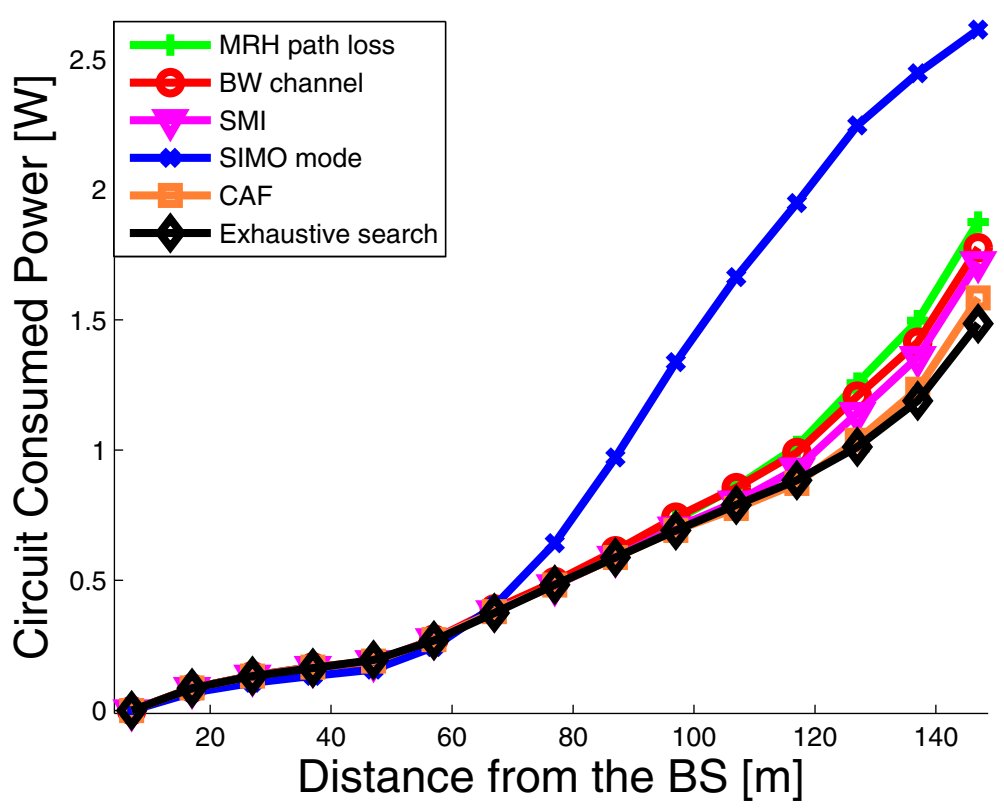

Figure 11 User circuit consumed power against distance from the BS for bit rate $\mathbf{9 2 0}$ kbps. Performance comparison when spatial multiplexing is implemented. 


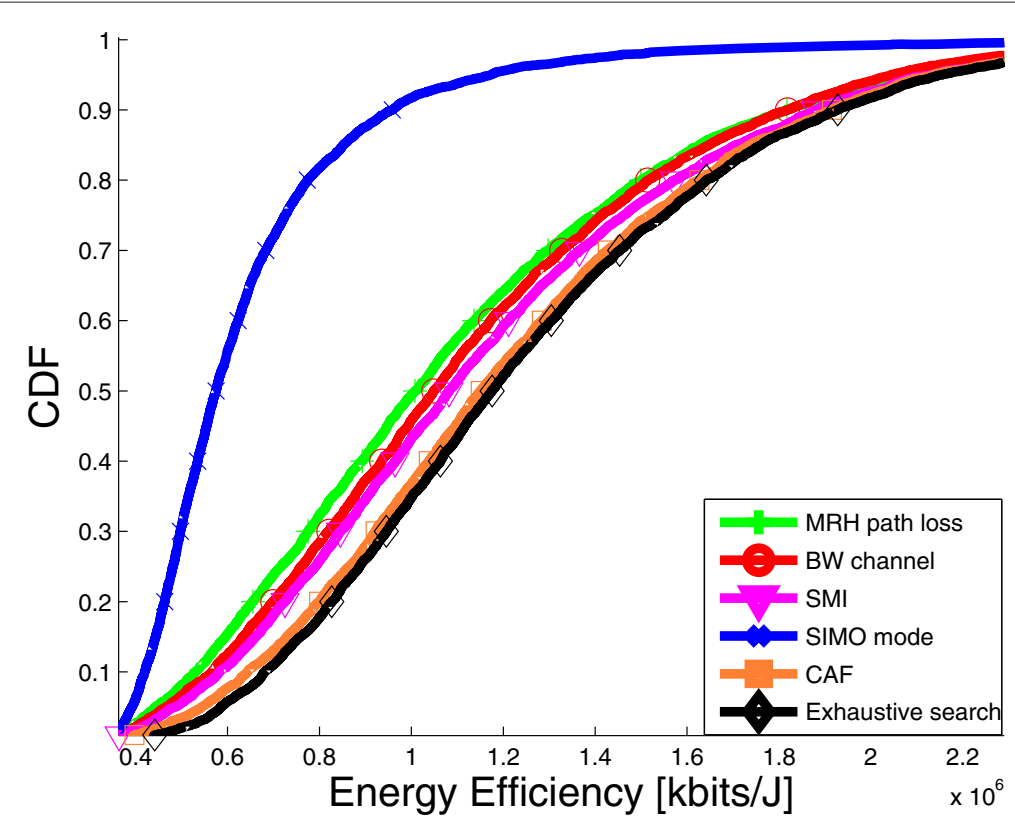

Figure 12 System energy efficiency when enhancing capacity. Performance comparison when spatial multiplexing is implemented.

in Section 6 when spatial multiplexing is implemented. We find that the centralized global optimum is $2 \%$ more energy efficient when contrasted to the CAF. Moreover, when comparing the CAF with the other distributed approaches, we see that the CAF method has improvements of $14 \%, 9 \%, 5 \%$, and $91 \%$ over the MRH, the BW, the SMI, and the baseline SIMO mode, respectively. Thereby, we can confirm that increasing the number of antennas in order to use a lower modulation order results in an energy efficient solution for the network.

To conclude our comparison, the complexity of the centralized global optimum approach is compared with the proposed CAF method. On one hand, for the CAF method, each MS- $n$ in the system has to evaluate each RS in its preferred subset of suitable candidates, $S_{n}$, by using Equation 29 or 30 depending on whether diversity or capacity are enhanced. Furthermore, each RS-r evaluates its preferred subset $S_{r}$ of RSs by using Equation 32 or 33. Big $\mathcal{O}$ notation is used to describe the growth rate of the both schemes. Thus, the system performs arithmetic operations with a complexity of $\mathcal{O}\left(\left|S_{n}\right|^{2}\right)$ and $\mathcal{O}\left(\left|S_{r}\right|^{2}\right)$ when candidate MSs or RS are ranked, respectively, where $|$.$| is defined as the cardinality of the subset.$ If we assume that $R \gg N$, the complexity of the candidate ranking process is bounded by the number of RSs in the system rather than by the number of MSs. Thereby, this will allow us to upper bound the complexity of the candidate ranking by $\mathcal{O}\left(\left|S_{n}\right|^{2}\right)$ operations. Moreover, forming the MS's preference list, $\iota_{n}$, Equation 31 requires a sorting operation which induces a complexity of $\mathcal{O}\left(\left|S_{n}\right| \log \left(\left|S_{n}\right|\right)\right)$ operations. Finally, the complexity of the decision-making, Algorithm 1 can be upper bounded by a binary search operation which requires a complexity of $\mathcal{O}\left(\log \left(\left|S_{n}\right|\right)\right)$ operations. Therefore, the dominant factor which determines the CAF scheme complexity will be the one with the largest exponent, thus the complexity of the method will be upper bounded by $\mathcal{O}\left(\left|S_{n}\right|^{2}\right)$ operations.

On the other hand, the centralized global optimum scheme is based on enumerating all possible alternatives for virtual MIMO coalition formation between the MS$n$ and its preferred subset of candidate RSs, $S_{n}$. This is done with the purpose of finding the optimal number of transmit antennas that would minimize the overall power consumption in the reverse link. Therefore, to guarantee that a given feasible solution is optimal, the solution should be compared with any other feasible solution. In general, an exhaustive search approach, where the number of elements is discrete, is considered $\mathcal{N P}$. complete [39]. A notable characteristic of $\mathcal{N} \mathcal{P}$-complete problems is that the required time to solve the problem increases very quickly as the size of the problem grows [39]. To implement the exhaustive search scheme, each MS in the system will evaluate the total number of possible combinations in its preferred subset of candidate RSs, $S_{n}$. Hence, the total number of possible combinations is computed by $\sum_{k=1}^{\left|S_{n}\right|}\left(\begin{array}{c}\left|S_{n}\right| \\ k\end{array}\right)$, where $\left(\begin{array}{c}\left|S_{n}\right| \\ k\end{array}\right)=$ $\frac{\left|S_{n}\right| !}{k !\left(\left|S_{n}\right|-k\right) !}$. Moreover, each combination is evaluated by Equation 20 or 21 depending if diversity or capacity are 


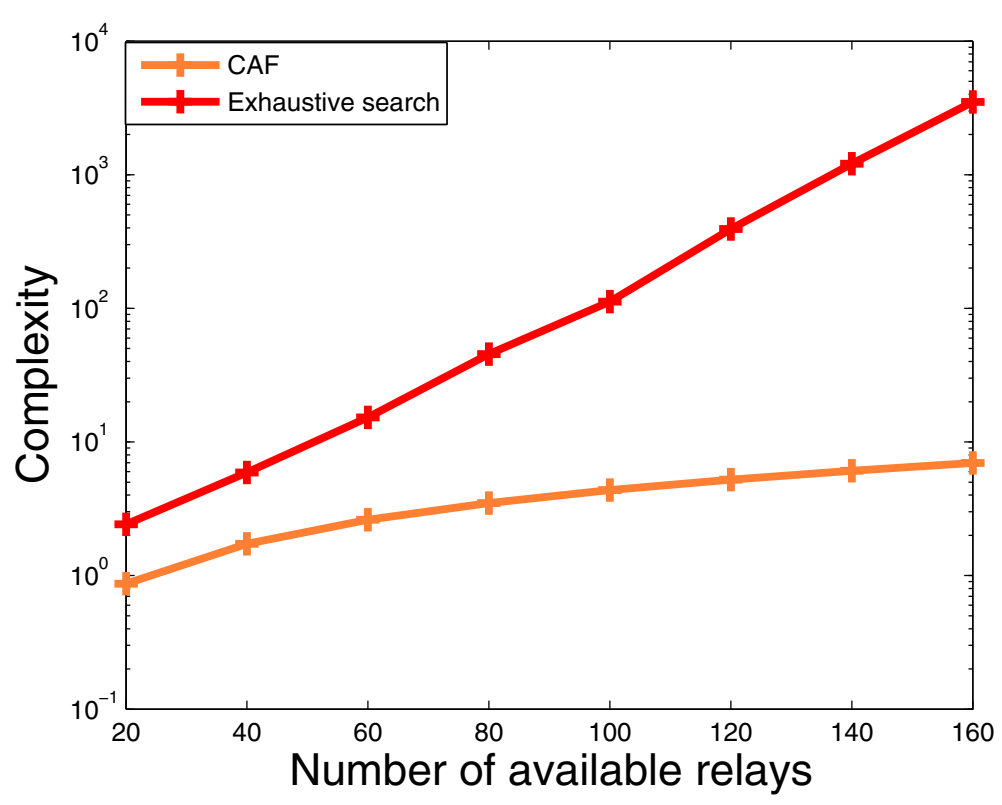

Figure 13 Complexity of the centralized optimum approach compared to the CAF method.

enhanced, respectively. Thus, this induces a complexity of $\mathcal{O}\left(\left(\sum_{k=1}^{\left|S_{m}\right|}\left(\begin{array}{c}\left|S_{m}\right| \\ k\end{array}\right)\right)^{2}\right)$ for the system. In addition, the complexity of both methods (exhaustive search and CAF) increases linearly with the number of MSs in the system, $N$. Hence, the exhaustive search method has a complexity of $\mathcal{O}\left(N \times\left(\sum_{k=1}^{\left|S_{n}\right|}\left(\begin{array}{c}\left|S_{n}\right| \\ k\end{array}\right)\right)^{2}\right)$ which is a higher order complexity when compared to the complexity of $\mathcal{O}\left(N \times\left|S_{n}\right|^{2}\right)$ for the CAF scheme. Furthermore, Figure 13 shows how the complexity of the system changes for both methods as the number of RSs increases in the system. It can be easily seen that as the number of RSs increases, the computational complexity of the exhaustive search increases exponentially, therefore it may not be a suitable solution to implement in real time systems.

\section{Conclusions}

In this paper, we considered a low complexity virtual MIMO coalition formation algorithm, which is based on game theory. The framework we proposed allows MSs to select the most suitable RSs providing the most power savings in the network. Thereby, we studied energy efficient coalition formation by using the concepts of diversity and spatial multiplexing, respectively. We have shown analytically and by simulation that increasing the number of transmit antennas is a more energy efficient solution for users close to the cell edge rather than for cell center users, when overall terminal power consumption is optimized. Furthermore, we have proven than the coalition formation algorithm we proposed is more energy efficient compared to the benchmark, the MRH path loss, the BW channel, and the SM framework with improvements of $58 \%, 15 \%, 10 \%$, and $5 \%$ for the spatial diversity case. When implementing spatial multiplexing, the CAF method has improvements of $14 \%, 9 \%, 5 \%$, and $91 \%$ over MRH, BW, SMI, and the baseline SIMO mode. It experiences only small performance losses of $10 \%$ and $2 \%$ when compared to an exhaustive search approach when implementing diversity and spatial multiplexing respectively. In addition, we presented a complexity analysis showing that the complexity of the method we proposed increases linearly as the number of RSs grows in the network. This is a much lower complexity when compared to the exponential growth of the exhaustive search scheme. Thus, the game-theory-based framework we proposed achieves a similar performance compared to a centralized scheme with a much lower complexity order. Hence, it may be a suitable energy-efficient solution for practical applications.

\section{Competing interests}

The authors declare that they have no competing interests.

\section{Acknowledgements}

Part of this work was done when Rodrigo Vaca was with the Institute of Digital Communications at the University of Edinburgh.

The work of Eitan Altman has been partially supported by the European Commission within the framework of the CONGAS project

FP7-ICT-2011-8-317672. 


\section{Author details}

${ }^{1}$ Scientific Division, Federal Police, 01110, Constituyentes 947, Mexico City, Mexico. ${ }^{2}$ The University of Edinburgh, Institute for Digital Communications, King's Buildings, Mayfield Road, EH93JL Edinburgh, UK. ${ }^{3}$ INRIA, BP93, 06902 Sophia Antipolis Cedex, France. ${ }^{4}$ Department of Electrical Engineering, Universidad Autónoma Metropolitana (UAM), 09340 Iztapalapa, Mexico City, Mexico.

\section{Received: 25 November 2014 Accepted: 23 February 2015} Published online: 26 March 2015

\section{References}

1. R Vaca, J Thompson, V Ramos, Non-cooperative uplink interference protection framework for fair and energy efficient orthogonal frequency division multiple access networks. IET Commun. 7(18), 2015-2025 (2013) doi:10.1049/iet-com.2013.0125

2. C Han, T Harrold, S Armour, I Krikidis, S Videv, PM Grant, H Haas, JS Thompson, I Ku, C-X Wang, TA Le, MR Nakhai, J Zhang, L Hanzo, Green radio: radio techniques to enable energy-efficient wireless networks. IEEE Commun. Mag. 49(6), 46-54 (2011). doi:10.1109/MCOM.2011.5783984

3. AR Jensen, M Lauridsen, P Mogensen, TB Sørensen, P Jensen, in Proceedings of the IEEE 76th Vehicular Technology Conference (VTC-Fall). LTE UE power consumption model for system level energy and performance optimization (IEEE, Quebec City, Canada, 2012), pp. 1-6. doi:10.1109/NTCFall.2012.6399281

4. R Vaca, J Thompson, V Ramos, in Proceedings of the IEEE 76th Vehicular Technology Conference (VTC-Fall). Uplink interference protection as a non-cooperative game over OFDMA networks (IEEE, Quebec City, Canada, 2012), pp. 1-5. doi:10.1109/NTCFall.2012.6399092

5. R Vaca, E Altman, J Thompson, V Ramos, in Proceedings of the 24th Annual IEEE International Symposium on Personal Indoor and Mobile Radio Communications (PIMRC). A stable marriage framework for distributed virtual MIMO coalition formation (IEEE, London, UK, 2013), pp. 2722-2727. doi:10.1109/PIMRC.2013.6666606

6. R Vaca, J Thompson, E Altman, V Ramos, in Proceedings of the IEEE INFOCOM Workshop on Green Cognitive Communications and Computing Networks. A game theory framework for a distributed and energy efficient bandwidth expansion process (IEEE, Toronto, Canada, 2014), pp. 712-717. doi:10.1109/INFCOMW.2014.6849318

7. A Paulraj, R Nabar, D Gore, Introduction to Space Time Wireless Communications. (Cambridge University Press, Cambridge UK, 2003)

8. J Jiang, M Dianati, M Imran, Y Chen, in Proceedings of the IEEE 76th Vehicular Technology Conference (VTC-Fall). Energy efficiency and optimal power allocation in virtual-MIMO systems (IEEE Quebec City, Canada, 2012), pp. 1-6. doi:10.1109/NTCFall.2012.6399002

9. S Sesia, I Toufik, M Bake, LTE-the UMTS Long Term Evolution from Theory to Practice. (Wiley, Chichester, USA, 2009)

10. Z Han, D Niyato, T Başar, A Hjørungnes, Game Theory in Wireless and Communication Networks. (Cambridge University Press, Cambridge, UK, 2012)

11. G Quer, F Librino, L Canzian, L Badia, M Zorzi, Inter-network cooperation exploiting game theory and bayesian networks. IEEE Trans. Commun. 61(10), 4310-4321 (2013). doi:10.1109/TCOMM.2013.082813.120710

12. S Hussain, A Azim, J Park, Energy efficient virtual MIMO communication for wireless sensor networks. Telecommun. Syst. 42(1-2), 139-149 (2009). doi:10.1007/s11235-009-9176-7

13. W Saad, Z Han, M Debbah, A Hjorungnes, T Başar, Coalitional game theory for communication networks. IEEE Signal Process. Mag. 26(5), 77-97 (2009). doi:10.1109/MSP.2009.000000

14. F Pantisano, M Bennis, W Saad, R Verdone, M Latva-aho, in Proceedings of the IEEE Wireless Communications and Networking Conference (WCNC). Coalition formation games for femtocell interference management: a recursive core approach (IEEE, Cancun, Mexico, 2011), pp. 1161-1166. doi:10.1109/WCNC.2011.5779295

15. AS Ibrahim, AK Sadek, W Su, KJR Liu, Cooperative communications with relay-selection: when to cooperate and whom to cooperate with? IEEE Trans. Wireless Commun. 7(7), 2814-2827 (2008). doi:10.1109/TWC 2008.070176
16. EG Larsson, EA Jorswieck, Competition versus cooperation on the MISO interference channel. IEEE J. Selected Areas Commun. 26(7), 1059-1069 (2008). doi:10.1109/JSAC.2008.080904

17. K Yazdi, E Gammal, P Schitner, in Allerton Conference on Communications, Control and Computing. On the design of cooperative transmission schemes, (2003)

18. N Jindal, U Mitra, A Goldsmith, in International Symposium on Information Theory (ISIT). Capacity of ad-hoc networks with node cooperation (IEEE, 2004), p. 269. doi:10.1109/ISIT.2004.1365306

19. S Cui, A Goldsmith, A Bahai, Energy efficiency of MIMO and cooperative MIMO techniques in sensor networks. IEEE J. Selected Areas Commun. 22(6), 1089-1098 (2004). doi:10.1109/JSAC.2004.830916

20. Z Han, D Niyato, W Saad, T Başar, A Hjørungnes, Game Theory in Wireless and Communication Networks. (Cambridge University Press, Cambridge, UK, 2012)

21. Z Han, R Liu, Fair multiuser channel allocation for OFDMA networks using Nash bargaining solutions and coalitions. IEEE Trans. Commun. 53(8), 1366-1376 (2005). doi:10.1109/TCOMM.2005.852826

22. W Saad, Z Han, M Debbah, A distributed coalition formation framework for fair user cooperation in wireless networks. IEEE Trans. Wireless Commun. 8(9), 4580-4593 (2009). doi:10.1109/TWC.2009.080522

23. H Burchardt, S Sinanovic, Z Bharucha, H Haas, Distributed and autonomous resource and power allocation for wireless networks. IEEE Trans. Commun. 61(7), 2758-2771 (2013). doi:10.1109/TCOMM.2013.053013.120916

24. WA Prasetyo, H Lu, H Nikookar, in Proceedings of the 41st European Microwave Conference (EuMC). Optimal relay selection and power allocation using game theory for cooperative wireless networks with interference. IEEE (IEEE, Manchester, UK, 2011), pp. 37-40

25. S Fujio, D Kimura, in Proceedings of the IEEE 24th International Symposium on Personal Indoor and Mobile Radio Communications (PIMRC). Energy saving effect of solar powered repeaters for cellular mobile systems. IEEE (IEEE London, UK, 2013), pp. 2841-2845. doi:10.1109/PIMRC.2013.6666631

26. J Laneman, D Tse, G Wornell, Cooperative diversity in wireless networks: efficient protocols and outage behavior. IEEE Trans. Inf. Theory. 50(12), 3062-3080 (2004). doi:10.1109/TIT.2004.838089

27. H Burchardt, Z Bharucha, G Auer, H Haas, Uplink interference protection and scheduling for energy efficient OFDMA networks. EURASIP J. Wireless Commun. Netw. 2012(180) (2012). doi:10.1186/1687-1499-2012-180

28. H Dai, in Proceedings of the IEEE International Conference on Acoustics, Speech and Signal Processing (ICASSP). Distributed versus co-located MIMO systems with correlated fading and shadowing, vol. 4 (IEEE, Toulouse, France, 2006), pp. 561-564. doi:10.1109/ICASSP.2006.1661030

29. Q Li, G Li, W Lee, M-I Lee, D Mazzarese, B Clerckx, Z Li, MIMO techniques in WiMAX and LTE: a feature overview. IEEE Commun. Mag. 48(5), 86-92 (2010). doi:10.1109/MCOM.2010.5458368

30. T Cover, J Thomas, Elements of Information Theory. (Wiley-Interscience, Hoboken, NJ, USA, 1991)

31. K Bobae, K Cholho, L Jongsoo, A dual-mode power amplifier with on-chip switch bias control circuits for LTE handsets. IEEE Trans. Circuits Syst. 58(12), 857-861 (2011). doi:10.1109/TCSII.2011.2172528

32. D Gale, M Sotomayor, Some remarks on the stable matching problem. Discrete Appl. Math. 11(3), 223-232 (1985) doi:10.1016/0166-218X(85)90074-5

33. A Roth, E Peranson, The redesign of the matching market for American physicians: some engineering aspects of economic design. Am. Econ. Rev. 89(4), 748-780 (1999). doi:10.1257/aer.89.4.748

34. K Iwama, S Miyazaki, in Proceedings of the International Conference on Informatics Research for Development of Knowledge Society Infrastructure. A survey of the stable marriage problem and its variants (IEEE, Kyoto, Japan, 2008), pp. 131-136. doi:10.1109/ICKS.2008.7

35. S Sanayei, A Nosratinia, Antenna selection in MIMO systems. IEEE Commun. Mag. 42(10), 68-73 (2004). doi:10.1109/MCOM.2004.1341263

36. D Gale, LS Shapley, College admissions and the stability of marriage. Am. Math. Mon. 69(1), 9-15 (1962)

37. V Sreng, $\mathrm{H}$ Yanikomeroglu, D Falconer, in Proceedings of the IEEE 58th Vehicular Technology Conference (VTC-Fall). Relayer selection strategies in 
cellular networks with peer-to-peer relaying (IEEE, 2003), pp. 1949-1953. doi:10.1109/NETECF.2003.1285365

38. EG Larsson, EA Jorswieck, J Lindblom, R Mochaourab, Game theory and the flat-fading gaussian interference channel. IEEE Signal Process. Mag. 26(5), 18-27 (2009). doi:10.1109/MSP.2009.933370

39. Z Han, KJR Liu, Resource Allocation for Wireless Networks. (Cambridge University Press, Cambridge, UK, 2008)

Submit your manuscript to a SpringerOpen ${ }^{\circ}$ journal and benefit from:

- Convenient online submission

- Rigorous peer review

- Immediate publication on acceptance

- Open access: articles freely available online

- High visibility within the field

- Retaining the copyright to your article

Submit your next manuscript at $\boldsymbol{\wedge}$ springeropen.com 